\title{
Dynamic binding mode of a Synaptotagmin-1-SNARE complex in solution
}

\author{
Kyle D Brewer ${ }^{1-3}$, Taulant Bacaj ${ }^{4,5}$, Andrea Cavalli6,7, Carlo Camilloni ${ }^{7}$, James D Swarbrick ${ }^{8}$, Jin Liu ${ }^{1-3,9}$, \\ Amy Zhou ${ }^{1-3}$, Peng Zhou ${ }^{4,5}$, Nicholas Barlow ${ }^{8}$, Junjie Xu ${ }^{1-3}$, Alpay B Seven ${ }^{1-3}$, Eric A Prinslow ${ }^{1-3}$, \\ Rashmi Voleti ${ }^{1-3}$, Daniel Häussinger ${ }^{10}$, Alexandre M J J Bonvin ${ }^{11}$, Diana R Tomchick ${ }^{1,2}$, Michele Vendruscolo ${ }^{7}$, \\ Bim Graham ${ }^{8}$, Thomas C Südhof ${ }^{4,5}$ \& Josep Rizo ${ }^{1-3}$
}

\begin{abstract}
Rapid neurotransmitter release depends on the $\mathrm{Ca}^{2+}$ sensor Synaptotagmin-1 (Syt1) and the SNARE complex formed by synaptobrevin, syntaxin-1 and SNAP-25. How Syt1 triggers release has been unclear, partly because elucidating high-resolution structures of Syt1-SNARE complexes has been challenging. An NMR approach based on lanthanide-induced pseudocontact shifts now reveals a dynamic binding mode in which basic residues in the concave side of the Syt $1 \mathrm{C}_{2} \mathrm{~B}$-domain $\beta$-sandwich interact with a polyacidic region of the SNARE complex formed by syntaxin-1 and SNAP-25. The physiological relevance of this dynamic structural model is supported by mutations in basic residues of Syt1 that markedly impair SNARE-complex binding in vitro and Syt1 function in neurons. Mutations with milder effects on binding have correspondingly milder effects on Syt1 function. Our results support a model whereby dynamic interaction facilitates cooperation between Syt1 and the SNAREs in inducing membrane fusion.
\end{abstract}

Neurotransmitter release is governed by a sophisticated protein machinery ${ }^{1,2}$. Central components of this machinery are the SNAREs synaptobrevin, syntaxin-1 and SNAP-25, which form a tight fourhelix bundle $\mathrm{e}^{3,4}$ that brings the synaptic vesicle and plasma membranes together and is key for membrane fusion ${ }^{5}$ (schematic in Supplementary Fig. 1a). $\mathrm{Ca}^{2+}$ triggering of fast neurotransmitter release is executed by Syt1 (ref. 6) via its two $\mathrm{C}_{2}$ domains. The $\mathrm{C}_{2} \mathrm{~A}$ and $\mathrm{C}_{2} \mathrm{~B}$ domains bind multiple $\mathrm{Ca}^{2+}$ ions through loops at the top of $\beta$-sandwich structures $^{7-9}$, and $\mathrm{Ca}^{2+}$-dependent membrane binding through these loops is key for Syt 1 function ${ }^{6}$. $\mathrm{Ca}^{2+}$ binding to the $\mathrm{C}_{2} \mathrm{~B}$ domain appears to have a predominant role in release ${ }^{10}$, which may arise from the ability of $\mathrm{C}_{2} \mathrm{~B}$ to bind simultaneously to two membranes ${ }^{11,12}$. The function of Syt 1 in release also depends on interactions with the SNAREs ${ }^{13}$ and is tightly coupled to complexins ${ }^{14-16}$, small soluble proteins with active and inhibitory roles in release ${ }^{17-19}$. Complexins bind to the SNARE complex through a central $\alpha$-helix, and they also contain an accessory $\alpha$-helix ${ }^{20}$ (Supplementary Fig. 1a) that inhibits release ${ }^{19,21}$, probably because of repulsion with the membranes ${ }^{22}$.

These and other advances have led to the reconstitution of synaptic-vesicle fusion with eight central components of the release machinery ${ }^{23}$, but fundamental questions remain about the mechanism of release. This uncertainty arises in part from the lack of high-resolution structures of Syt1-SNARE complexes. Thus, it is unclear which of the diverse Syt1-SNARE interactions reported ${ }^{24}$ are physiologically relevant. Syt1 interacts with isolated syntaxin-1 and SNAP-25 (refs. 25-28), although it is unknown whether SNARE-complex binding involves these interactions, and distinct regions of SNAP-25 have been implicated in such binding ${ }^{29,30}$. Some studies have reported that SNARE-complex binding involves a polybasic region on the side of $\mathrm{C}_{2} \mathrm{~B}^{30-32}$ (Fig. 1a), but other studies have implicated the bottom of $\mathrm{C}_{2} \mathrm{~B}^{33}$ or other weak binding sites of Syt 1 that contribute to aggregation with the SNARE complex ${ }^{34}$. It is also puzzling that Syt 1 and a complexin-I fragment spanning the central and accessory $\alpha$-helices (CpxI(26-83)) bind simultaneously to the SNARE complex in solution and yet compete for binding to SNARE complexes on membranes ${ }^{35}$.

The study described here results from 15 years of attempts to elucidate the structure of Syt1-SNARE complexes and used sensitive NMR methods ${ }^{36}$ to measure lanthanide-induced pseudocontact shifts (PCSs) ${ }^{37}$ induced on Syt 1 fragments by lanthanide probes attached to the SNARE complex. Our data delineate a dynamic structure in which binding is mediated by adjacent acidic regions from syntaxin-1 and SNAP-25, and by the basic concave side of the Syt $1 \mathrm{C}_{2} \mathrm{~B}$-domain $\beta$-sandwich, including residues from the polybasic region. The physiological relevance of this dynamic structure is supported by the parallel effects caused by mutations in basic residues of the $\mathrm{C}_{2} \mathrm{~B}$ domain on SNARE-complex binding in vitro and on Syt1 function in neurons. Moreover, the observed Syt1-SNARE-complex binding

${ }^{1}$ Department of Biophysics, University of Texas Southwestern Medical Center, Dallas, Texas, USA. ${ }^{2}$ Department of Biochemistry, University of Texas Southwestern Medical Center, Dallas, Texas, USA. ${ }^{3}$ Department of Pharmacology, University of Texas Southwestern Medical Center, Dallas, Texas, USA. ${ }^{4}$ Department of Molecular and Cellular Physiology, Stanford University Medical School, Stanford, California, USA. ${ }^{5}$ Howard Hughes Medical Institute, Stanford University Medical School, Stanford, California, USA. ${ }^{6}$ Institute for Research in Biomedicine, Bellinzona, Switzerland. ${ }^{7}$ Department of Chemistry, University of Cambridge, Cambridge, UK. ${ }^{8}$ Monash Institute of Pharmaceutical Sciences, Monash University, Parkville, Victoria, Australia. ${ }^{9}$ Department of Chemistry, Southern Methodist University, Dallas, Texas, USA. ${ }^{10}$ Department of Chemistry, University of Basel, Basel, Switzerland. ${ }^{11}$ Bijvoet Center for Biomolecular Research, Faculty of Science, Utrecht University, Utrecht, the Netherlands. Correspondence should be addressed to J.R. (jose@arnie.swmed.edu). 
Figure 1 A polybasic region of the Syt 1 $\mathrm{C}_{2} \mathrm{~B}$ domain binds to the SNARE complex. (a) Ribbon diagram of the Syt $1 \mathrm{C}_{2} \mathrm{~B}$ domain showing the side chains that form the polybasic region, other basic residues that were mutated in this study, and Val283, Arg398 and Arg399 at the bottom of the domain. Basic residues are colored in blue and Val283 in green. $\mathrm{Ca}^{2+}$ ions are represented by yellow spheres. $\mathrm{N}$ and $\mathrm{C}$ represent the $\mathrm{N}$ and $\mathrm{C}$ termini, respectively. (b) ${ }^{1} \mathrm{H}-{ }^{15} \mathrm{~N}$ TROSY HSQC spectra of ${ }^{2} \mathrm{H}-{ }^{15} \mathrm{~N}-\mathrm{C}_{2} \mathrm{AB}$ $(50 \mu \mathrm{M})$ in the absence (black contours) and presence of 10, 20 or $40 \mu \mathrm{M}$ SNARE complex (green, red and blue contours, respectively). (c) Expansions of the regions corresponding to the G175, V283, R322, K325, K326 and K327 cross-peaks in the spectra shown in $\mathbf{b}$.

mode potentially explains why Syt 1 competes with CpxI(26-83) for binding to the SNARE complex on membranes but not in solution. Although our results need to be interpreted with caution (as discussed below), they are consistent with a model in which binding of Syt 1 to the SNARE complex places the Syt $1 \mathrm{C}_{2} \mathrm{~B}$ domain in an ideal position to release the inhibition caused by the CpxI accessory $\alpha$-helix and to bridge the two membranes, thus allowing Syt 1 to cooperate with the SNAREs in membrane fusion.

\section{RESULTS}

The SNARE complex binds to the $\mathrm{C}_{2} \mathrm{~B}$-domain polybasic region To overcome the tendency of Syt1-SNARE complexes to aggregate in the presence of $\mathrm{Ca}^{2+}$, we used a Syt 1 fragment containing both $\mathrm{C}_{2}$ domains $\left(\mathrm{C}_{2} \mathrm{AB}\right)$ and exhibiting improved behavior ${ }^{35}$, together with a buffer containing $125 \mathrm{mM}$ thiocyanate, a chaotropic anion that disrupts nonspecific protein binding ${ }^{38,39}$ (Supplementary Note 1). The SNARE complex induced shifts in specific cross-peaks of the ${ }^{1} \mathrm{H}-{ }^{15} \mathrm{~N}$ transverse relaxation optimized spectroscopy (TROSY)-HSQC spectrum of ${ }^{2} \mathrm{H}-{ }^{15} \mathrm{~N}$-labeled $\mathrm{C}_{2} \mathrm{AB}$ (Fig. 1b,c), most of which correspond to the
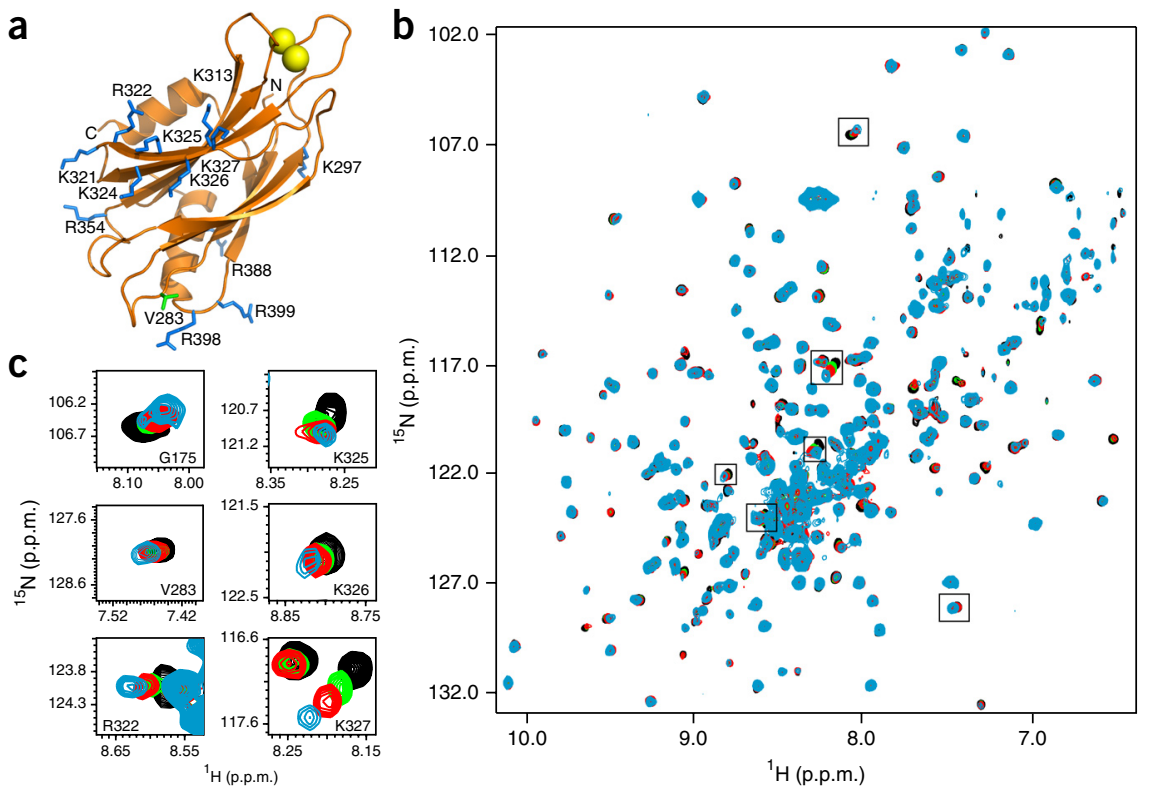

$\mathrm{C}_{2} \mathrm{~B}$ polybasic region (Fig. 1a). Thus, the polybasic region constitutes the primary binding site on $\mathrm{C}_{2} \mathrm{AB}$ for the SNARE complex under these conditions containing $125 \mathrm{mM}$ KSCN. The cross-peaks of G175 (in a $\mathrm{C}_{2} \mathrm{~A} \mathrm{Ca}^{2+}$-binding loop) and V283 (at the bottom of $\mathrm{C}_{2} \mathrm{~B}$ ) also exhibited small shifts (Fig. 1). Because these regions provide additional, weaker binding sites that contribute to the aggregation of Syt1-SNARE complexes $^{34}$, these shifts suggest that these regions still bind to the SNARE complex, albeit very weakly under our conditions. The cross-peak shifts in the polybasic region are also small (Fig. 1c) because binding is mediated by ionic interactions between flexible side chains (discussed below). Indeed, reverse experiments with ${ }^{2} \mathrm{H}_{-}{ }^{15} \mathrm{~N}$-labeled SNARE complexes did not reveal substantial shifts (Supplementary Fig. 1). The limited solubility of the SNARE complex hindered the assignment of its side chain resonances ${ }^{20}$ and hence the analysis of perturbations in side chain chemical shifts to map the Syt1-binding site.
Figure $2 \Delta \chi$ tensors defined by the PCSs induced in the SNARE complex by $\mathrm{Dy}^{3+}{ }_{-} \mathrm{C} 2$ labels on residue 166 or 41 of SNAP-25. (a,d) ${ }^{1} \mathrm{H}-{ }^{15} \mathrm{~N}$ TROSY-HSQC spectra of SNARE-complex samples containing ${ }^{2} \mathrm{H}-15 \mathrm{~N}$-syntaxin-1 (a) or ${ }^{2} \mathrm{H}-{ }^{15} \mathrm{~N}$-synaptobrevin (d) and $\mathrm{Dy}^{3+}-\mathrm{C} 2$ labels on residue 166 (a) or 41 (d) of SNAP-25 before (red contours) or after (black contours) removal of the tag. Blue lines connect selected corresponding red and black cross-peaks, illustrating the observed PCSs. (b,e) Correlation between experimental PCSs measured with $\mathrm{Dy}^{3+}{ }_{-} \mathrm{C} 2$ labels on residue 166 (b) or 41 (e) of SNAP-25 and PCSs calculated with the $\Delta \chi$ tensors derived from the experimental values. Correlation coefficients $(r)$ and slopes $(m)$ are indicated. The values obtained for the axial and rhombic components of the tensor, $\Delta \chi_{\mathrm{ax}}$ and $\Delta \chi_{\mathrm{rh},}\left(10^{-32} \mathrm{~m}^{3}\right)$ are 35.1 and 2.9 , respectively, for the SC166 tensor (b) and 15.9 and 6.7, respectively, for the SC41 tensor (e). $(\mathbf{c}, \mathbf{f})$ Ribbon diagrams of the SNARE complex (yellow, syntaxin; red, synaptobrevin; blue and green, SNAP-25 N-terminal and C-terminal SNARE motifs, respectively) with isosurfaces representing regions with positive (blue) and negative (red) PCSs, contoured at \pm 0.8 p.p.m. with the SC166 (c) and SC41 (f) tensors. The tensor centers are indicated with black spheres. The same color coding for the SNAREs is used in all figures.
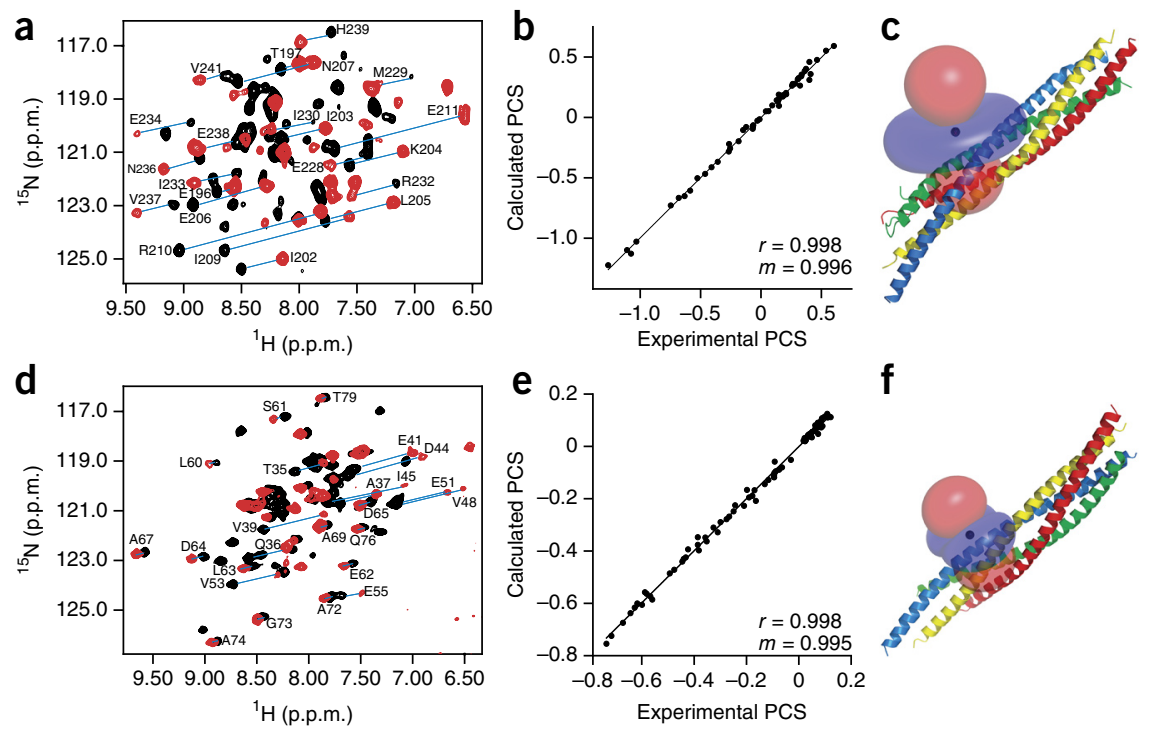


\section{Pseudocontact shifts from the SNARE complex to Syt1 $C_{2} B$}

The existence of sparsely populated states hindered structural analyses of $\mathrm{C}_{2} \mathrm{AB}-\mathrm{SNARE}$ complexes with paramagnetic relaxation effects but is less of an obstacle for studies with lanthanide-induced $\mathrm{PCSs}^{37}$ (Supplementary Note 1). After attempts for several years with different lanthanide-chelating tags placed at diverse positions (Supplementary Note 1), we used SNARE complexes labeled with a 1,4,7,10 tetraazacyclododecane-tetraacetic acid (DOTA)-based tag called C2 (no relation to the term $\mathrm{C}_{2}$ domain) ${ }^{40,41}$ loaded with $\mathrm{Dy}^{3+}$. SNARE complexes labeled with $\mathrm{Dy}^{3+}{ }_{-} \mathrm{C} 2$ on residues 41 or 166 of SNAP-25 (below referred to as SC41Dy or SC166Dy, respectively) exhibited strong PCSs that could be fit to unique anisotropic magnetic susceptibility tensors ( $\Delta \chi$ tensors; Fig. 2 ).

To analyze the Syt1-SNARE -complex binding mode, we used ${ }^{15} \mathrm{~N}-{ }^{2} \mathrm{H}$-labeled Syt 1 fragments specifically ${ }^{1} \mathrm{H}_{-}{ }^{13} \mathrm{C}$-labeled at isoleucine, leucine and valine methyl groups $\left({ }^{15} \mathrm{~N}-{ }^{2} \mathrm{H}-\mathrm{ILV}-{ }^{13} \mathrm{CH}_{3}-\right.$ labeling) for optimal relaxation properties ${ }^{36}$. Most of the PCSs induced by SC41Dy or SC166Dy on Syt1 $\mathrm{C}_{2} \mathrm{AB}$ were observed for cross-peaks from $\mathrm{C}_{2} \mathrm{~B}$, and very similar PCSs were observed when, instead of $\mathrm{C}_{2} \mathrm{AB}$, we used the isolated $\mathrm{C}_{2} \mathrm{~B}$ domain or the $\mathrm{C}_{2} \mathrm{~B}$ domain with an $\mathrm{R} 398 \mathrm{Q}$ R399Q mutation ${ }^{34}$ that hinders aggregation with the SNARE complex (Fig. 3a,b, Supplementary Fig. 2a,b,d,e and Online Methods). Because the latter yielded the best-quality data, and Arg398 and Arg399 might mediate binding modes that promote aggregation, we performed structural analyses with PCS data obtained from ${ }^{1} \mathrm{H}_{-}{ }^{15} \mathrm{~N}$ TROSY-TROSY HSQC and ${ }^{1} \mathrm{H}_{-}{ }^{13} \mathrm{C}$ heteronuclear multiple quantum coherence (HMQC) spectra of ${ }^{15} \mathrm{~N}-{ }^{2} \mathrm{H}-\mathrm{ILV}-{ }^{13} \mathrm{CH}_{3}-\mathrm{C}_{2} \mathrm{~B}$ domain bearing the R398Q R399Q mutation (below referred to as $\mathrm{C}_{2} \mathrm{~B}$ for simplicity). These included 149 and 151 PCSs induced by SC41Dy or SC166Dy, respectively, on the $\mathrm{C}_{2} \mathrm{~B}$ domain. $\mathrm{C}_{2} \mathrm{~B}$ binding did not affect the PCSs within SC166Dy but caused

Figure 3 PCSs induced by SC166Dy and SC41Dy on the Syt $1 \mathrm{C}_{2} \mathrm{~B}$ domain. $(\mathbf{a}, \mathbf{b}){ }^{1} \mathrm{H}_{-}{ }^{13} \mathrm{C}$ HMQC spectra of $30 \mu \mathrm{M}{ }^{15} \mathrm{~N}-{ }^{2} \mathrm{H}-I \mathrm{LV}-{ }^{13} \mathrm{CH}_{3}-\mathrm{C}_{2} \mathrm{~B}$ R398Q R399Q mutant in the presence of $30 \mu \mathrm{M}$ SC166Dy (a) or SC41Dy (b) before (red contours) or after (black contours) tag removal. (c,d) Ribbon diagrams of Syt $1 C_{2} B$ showing PCSs induced by SC166Dy (c) or SC41Dy (d). Amide hydrogens and methyl carbons are shown as spheres color coded according to the measured PCSs (dark blue, $>0.06$ p.p.m.; blue, 0.04 to 0.06 p.p.m.; cyan, 0.02 to 0.04 p.p.m.; pale cyan, 0.008 to 0.02 ppm; red, -0.04 to -0.06 p.p.m.; salmon, -0.02 to -0.04 p.p.m.; light pink, -0.008 to -0.02 p.p.m.). Yellow spheres represent $\mathrm{Ca}^{2+}$ ions. $(e, f)$ Models of $\mathrm{C}_{2} \mathrm{~B}$ bound to the SNARE complex built manually to match the $\mathrm{C}_{2} \mathrm{~B}$ PCSs with the SC166 (e) and SC41 (f) tensors represented by isosurfaces (as in Fig. 2c,f). $(\mathbf{g}-\mathbf{j})$ Correlations between experimental PCSs induced on $\mathrm{C}_{2} \mathrm{~B}$ by SC166Dy (g,h) or SC41Dy $(\mathbf{i}, \mathbf{j})$ and PCSs calculated with the 166- and 41-manual models with the optimized SC166 (g) and SC41 (i) tensors (illustrated in Fig. $\mathbf{2 c}$,f, respectively) or slightly modified tensors $(\mathbf{h}, \mathbf{j})$ (Supplementary Note 7). Correlation coefficients ( $r$ ) and slopes $(m)$ are indicated. slight alterations of PCSs within SC41Dy (5-20\%; Supplementary Fig. 2f,g), thus showing that $\mathrm{C}_{2} \mathrm{~B}$ does not contact residue 166 but is close to residue 41 . Nevertheless, we analyzed the SC41Dy data to examine the consistency with the SC166Dy data. We also note that addition of CpxI caused either no changes or very small changes in the PCSs induced by SC166Dy (Supplementary Fig. 2c), thus indicating that the binding sites for Syt 1 and CpxI on the SNARE complex in solution are distinct but proximal.

\section{Dynamic nature of the Syt1-SNARE complex in solution}

We could not apply standard methods used for stable protein complexes $^{37}$ to derive a structure of the $\mathrm{C}_{2} \mathrm{~B}-\mathrm{SNARE}$ complex from the PCSs induced on $\mathrm{C}_{2} \mathrm{~B}$ by SC166Dy (Online Methods and Supplementary Fig. 3) or SC41Dy. Because these PCSs exhibit defined patterns of positive-negative values when mapped onto the structure of $\mathrm{C}_{2} \mathrm{~B}$ (Fig. 3c,d), we manually matched these patterns with the positive-negative lobes of the $\Delta \chi$ tensors obtained from the PCSs measured within SC166Dy and SC41Dy (referred to as SC166 and SC41 tensors, respectively; Fig. 2a) while keeping contact between $\mathrm{C}_{2} \mathrm{~B}$ and the SNAREs. The $\mathrm{C}_{2} \mathrm{~B}$ PCSs calculated with the resulting models (referred to as the 166- and 41-manual models, respectively; Fig. 3e,f) and the SC166 or SC41 tensors have reasonable correlations with the measured PCSs, albeit with large slopes (Fig. 3g,i).

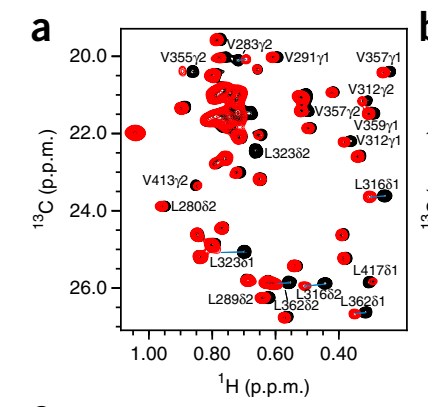

C
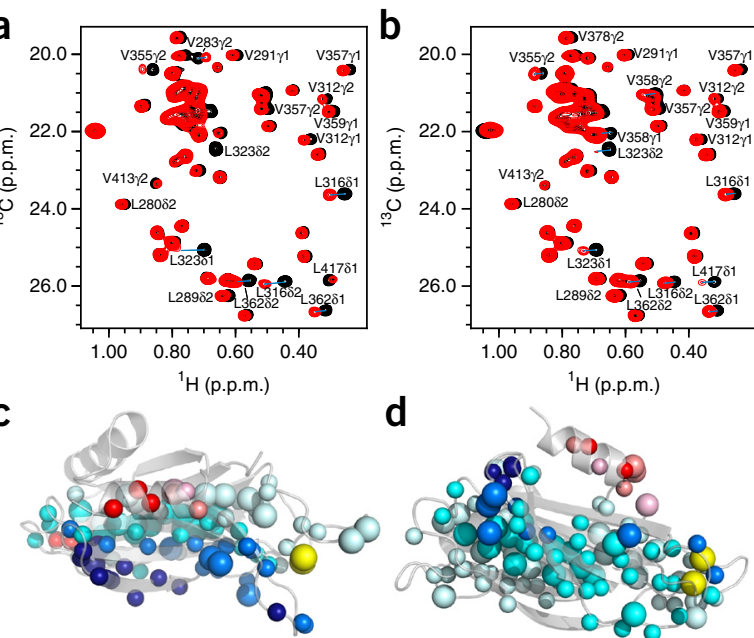

d
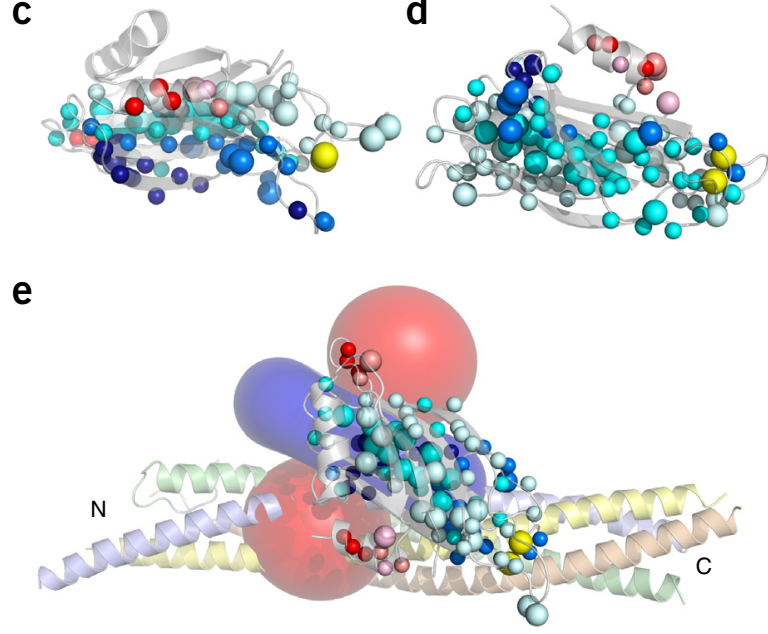

f

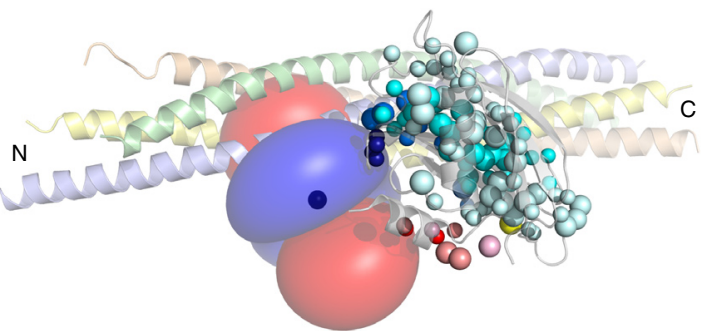

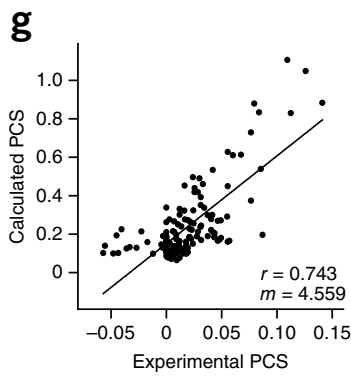
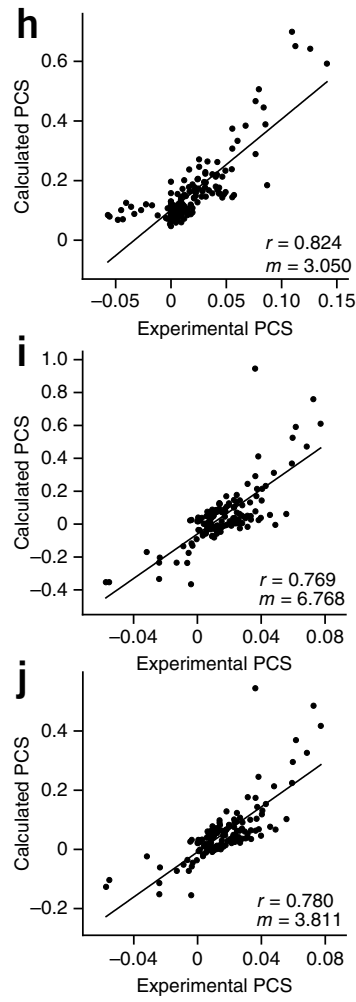
Figure 4 Analysis of the $\mathrm{C}_{2} \mathrm{~B}-\mathrm{SNARE}$ complex by MD simulations. (a) Ribbon diagrams of the SNARE complex and $\mathrm{C}_{2} \mathrm{~B}$ in the positions corresponding to the 166-manual model (gray), the 166-HADDOCK model (purple) and the 166-MD model (orange). (b) Ribbon diagram of the SNARE complex and stick models showing $\mathrm{C} \alpha$ traces of $\mathrm{C}_{2} \mathrm{~B}$ in a range of orientations visited during the $M D$ simulation started from the 166-HADDOCK model (purple). One of the structures from the end of the simulation (in orange) is represented in a and is referred to as the 166-MD model. (c) Ribbon diagrams of the SNARE complex and $\mathrm{C}_{2} \mathrm{~B}$ in the positions corresponding to the 166-MD model (orange) and the 41-manual model (cyan). (d) Ribbon diagram of the SNARE complex and stick models showing the $C \alpha$ traces of $C_{2} B$ in a range of representative orientations

visited during MD simulations incorporating chemical-shift restraints. The structure of the CpxI(26-83)-SNARE complex (PDB 1KIL) has been superimposed to show that $\mathrm{Cpxl}$ would bump into $\mathrm{C}_{2} \mathrm{~B}$ in some of the positions in the MD simulations. $\mathrm{N}$ represents the $\mathrm{N}$ terminus of the SNARE complex in a-d. $N$ and $C$ represent the $N$ and $C$ termini of $C p x I(26-83)$ in $\mathbf{d}$. (e,f) Correlations between experimental $C_{2} B$ PCSs induced by SC166Dy (e) or SC41Dy (f) and PCSs calculated as ensemble averages from different populations of structures from the 73 clusters visited during the chemical shift-restrained MD simulations. Correlation coefficients $(r)$ and slopes $(m)$ are indicated.
These slopes need to be interpreted with caution because they depend strongly on the position of the center of each tensor, which has some uncertainty $(\sim 4 \AA)$. Thus, varying the tensor centers within this range can yield smaller slopes with slightly improved correlations between measured and calculated PCSs (Fig. 3h,j).

The large slopes in Figure $\mathbf{3 g}-\mathbf{j}$ can be attributed to a highly dynamic structure in which the $\mathrm{C}_{2} \mathrm{~B}$ domain binds to the SNARE complex in multiple orientations at the same or nearby sites. This dynamic nature leads to averaging of the PCSs to smaller values than those expected for a static structure and is also manifested in the different shapes of the tensors derived from PCSs measured on $\mathrm{C}_{2} \mathrm{~B}$ and the SNARE complex (Supplementary Fig. 3a,b). Indeed, attempts to derive single $\mathrm{C}_{2} \mathrm{~B}-$ SNARE-complex structures consistent with the SC166Dy-induced PCSs with HADDOCK-PCS ${ }^{42}$ yielded structures in which $\mathrm{C}_{2} \mathrm{~B}$ was 'pushed away' from the center of the SC166 tensor, an expected effect of dynamic averaging of PCSs ${ }^{43}$ (illustrated in Fig. 4a for a representative structure; referred to as the 166-HADDOCK model). Moreover, the HADDOCK-PCS structures exhibited few salt bridges between $\mathrm{C}_{2} \mathrm{~B}$ and the SNAREs, and the pattern of positive-negative PCSs did not match the SC166 tensor lobes well (example in Supplementary Fig. 4b). Interestingly, in unrestrained molecular dynamics (MD) simulations started with the 166-HADDOCK model, $\mathrm{C}_{2} \mathrm{~B}$ moved naturally toward the position of the 166-manual model (Fig. 4a,b). A representative structure from the end of the simulation (referred to as the 166-MD model) exhibits abundant $\mathrm{C}_{2} \mathrm{~B}$-SNARE salt bridges (described below), a relatively good correlation between calculated and measured PCSs (Supplementary Fig. 4c) and a good match of positive-negative PCS patterns with the SC166 tensor lobes (Supplementary Fig. 4d). Notably, the position of the $\mathrm{C}_{2} \mathrm{~B}$ domain in the $166-\mathrm{MD}$ model is also close to that observed in the 41-manual model (Fig. 4c).

To explore the range of structures that form the ensemble of $\mathrm{C}_{2} \mathrm{~B}$-SNARE-complex binding modes, we performed extensive MD simulations with chemical-shift restraints ${ }^{44}$. Importantly, the 166-MD model and 41-manual model are located in the middle of the ensemble of structures visited during the simulations (Fig. 4d), thus showing a striking consistency with the PCS analysis. Calculation of $\mathrm{C}_{2} \mathrm{~B}$ PCSs for representative MD structures and optimization of population weights (Online Methods) yielded some degree of correlation between the population-averaged and measured PCSs, with slopes much closer to 1 than those obtained with individual models (Fig. 4e,f). The correlations are rather modest, indicating that additional structures are needed to fully account for the observed PCSs, but these results illustrate how conformational averaging decreases the calculated PCSs to values closer to those observed experimentally.

Overall, our analysis shows that the Syt $1 \mathrm{C}_{2} \mathrm{~B}-\mathrm{SNARE}$-complex binding mode is highly dynamic under our conditions. The 166-MD (or 166-manual) model and the 41-manual model can be considered to be representative structures located approximately at the center of the ensemble of binding modes. Differences between the two models could arise from slight perturbation of the binding ensemble caused by the tag in SC41Dy (described above), but, given the uncertainty of this analysis, the two models are quite similar (Fig. 4c) and verify the consistency of the two PCS data sets.

\section{The Syt1-SNARE-complex binding mode in solution}

We anticipate that the development of restrained MD simulations incorporating replica-averaged PCS restraints, as described for residual dipolar couplings ${ }^{45}$, will allow a more extensive exploration of the ensemble of $\mathrm{C}_{2} \mathrm{~B}$-SNARE-complex orientations to fit the PCS data better. Importantly, despite the relative uncertainty remaining about this ensemble, our results yield a clear picture of the nature of the Syt1-SNARE-complex interactions that predominate under our conditions, including models that can be used to probe the functional importance of these interactions and to elucidate how Syt1 and the SNAREs cooperate in triggering release.

The PCS data establish that the polybasic region of the $\mathrm{C}_{2} \mathrm{~B}$ domain binds to a polyacidic region formed by residues from syntaxin- 1 and SNAP-25 (examples in Fig. 5a-d), a result consistent with the high sensitivity of Syt1-SNARE-complex interactions to ionic strength ${ }^{14}$. The abundance of basic residues from $\mathrm{C}_{2} \mathrm{~B}$ and acidic residues from the SNAREs in the binding interface provide a natural explanation for the dynamic nature of Syt1-SNARE-complex interactions. Thus, any single structure would represent one of many binding modes existing in solution. Even with the caveat just mentioned, common features observed in the manual and MD models lead to defined predictions about which residues are most important for binding. A central feature 
Figure 5 The Syt $1 \mathrm{C}_{2} \mathrm{~B}-\mathrm{SNARE}$-complex binding mode. (a,b) Ribbon diagrams of the 166-MD model (a) and the 41-manual model (b), with $\mathrm{C}_{2} \mathrm{~B}$ shown in orange and $\mathrm{Ca}^{2+}$ ions represented by cyan spheres. Stick models show the side chains of basic (blue) and acidic (red) residues. Basic side chains from the polybasic strand and the concave side of $\mathrm{C}_{2} \mathrm{~B}$ are labeled. $(\mathbf{c}, \mathrm{d})$ Close-up views of the binding modes observed in the 166-MD model (c) and 41-MD model (d). Representation and color coding are as in $\mathbf{a}$ and $\mathbf{b}$. Selected basic and acidic side chains in the interfaces are labeled. (e) Ribbon diagrams showing the positions of $\mathrm{C}_{2} \mathrm{~B}$ in the 166-MD model (orange) and the 41-manual model (cyan) after superposition with the structure of the CpxI(26-83)-SNARE complex (PDB 1KIL). CpxI(26-83) is shown in pink (accessory helix) and gray (central helix). The dashed line represents a membrane surface and illustrates that binding of $\mathrm{C}_{2} \mathrm{~B}$ to a membrane would lead to steric and electrostatic repulsion of the $\mathrm{Cpxl}$ accessory helix with the membrane. $\mathrm{N}$ denotes $\mathrm{N}$ termini of the SNARE complex in $\mathbf{a}$ and $\mathbf{b}$ and of $\mathrm{CpxI}(26-83)$ and $\mathrm{C}_{2} \mathrm{~B}$ in e. $\mathrm{C}$ denotes $\mathrm{C}$ termini of $\mathrm{CpxI}(26-83)$ and of the SNARE complex in $\mathbf{e}$.

is that the concave surface of the $\mathrm{C}_{2} \mathrm{~B}$ $\beta$-sandwich is oriented toward the SNARE complex (Fig. 5a,b). Thus, although the polybasic $\beta$-strand of $C_{2} B$ (residues 321-327) was previously viewed as a functional unit, the observed orientation predicts key distinctions between the functional importance of basic side chains in this region. R322, K325 and K327 from this $\beta$-strand and K313 from the adjacent $\beta$-strand are oriented toward the SNARE complex and hence are likely to have critical roles in binding; in contrast, K324 and K326 are not directly oriented toward the SNARE complex and are predicted to have less prominent roles.

There is more variability in the residues of the SNARE complex that bind to $\mathrm{C}_{2} \mathrm{~B}$ in the different models. In the 166-MD model, the $\mathrm{C}_{2} \mathrm{~B}$ concave side exhibits multiple ionic interactions with an acidic region of syntaxin-1 (E224, E228, D231 and E234) but is also near an acidic region of SNAP-25 (D51, E52 and E55) (Fig. 5c). In the 41-manual model, $\mathrm{C}_{2} \mathrm{~B}$ interacts primarily with this SNAP-25 acidic region (Fig. 5b). A detailed analysis is not useful in this case because we built the model manually, but multiple structures from the chemical shift-restrained $\mathrm{MD}$ simulations placed $\mathrm{C}_{2} \mathrm{~B}$ in locations close to that observed in the 41-manual model (example in Supplementary Fig. 5; referred to as the 41-MD model) and illustrate how K313, R322, K325 and $\mathrm{K} 327$ of $\mathrm{C}_{2} \mathrm{~B}$ can establish multiple salt bridges with the SNAP-25 acidic region (Fig. 5d).

This analysis shows that the large number of charges in the $\mathrm{C}_{2} \mathrm{~B}$ polybasic concave side and the polyacidic region formed by syntaxin-1 and SNAP-25 allow formation of multiple salt bridges in many different orientations, explaining the dynamic nature of the binding mode. Importantly, our results also provide a clear explanation for the finding that Syt1 $\mathrm{C}_{2} \mathrm{AB}$ and $\mathrm{CpxI}(26-83)$ bind simultaneously to soluble SNARE complex but compete for binding to membrane-anchored SNARE complex ${ }^{35}$. Thus, the binding sites of
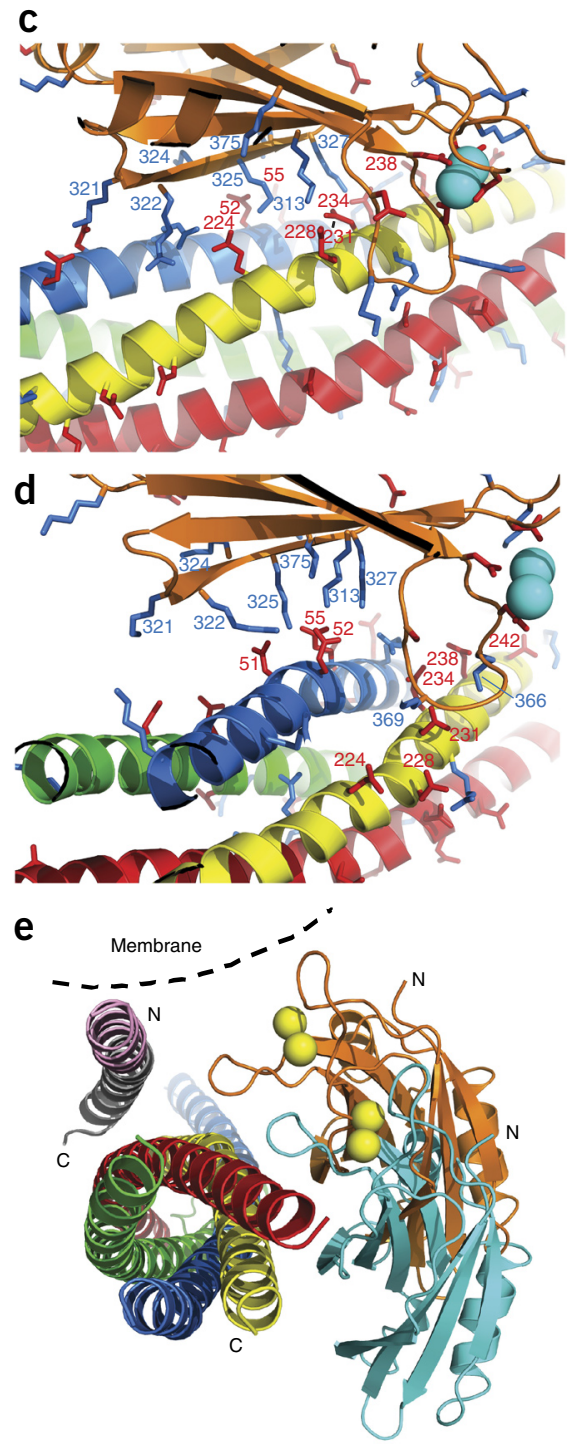

the SNARE complex for $\mathrm{CpxI}(26-83)$ and $\mathrm{C}_{2} \mathrm{~B}$ are distinct, allowing simultaneous binding in solution; however, $\mathrm{Ca}^{2+}$-induced binding of $\mathrm{C}_{2} \mathrm{~B}$ to a membrane would cause strong steric and electrostatic repulsion between the membrane and the CpxI accessory helix, both of which are negatively charged (Fig. 5e).

\section{Mutagenesis analysis of Syt1-SNARE-complex binding}

To test the Syt1-SNARE-complex binding mode derived from our PCS data, we used an assay ${ }^{46}$ that monitors the decrease in intensity of the strongest methyl resonance (SMR) in $1 \mathrm{D}^{13} \mathrm{C}$-edited ${ }^{1} \mathrm{H}$ NMR spectra of ${ }^{13} \mathrm{C}$-labeled $\mathrm{C}_{2} \mathrm{AB}$ upon binding to unlabeled SNARE complex (Fig. 6a). In initial experiments performed in the absence of KSCN, to extend a recent study ${ }^{34}$, we found that single-residue substitutions in the $\mathrm{C}_{2} \mathrm{~B}$ polybasic region did not alter binding strongly (Supplementary Fig. 6a,b). Given the large number of charges at the binding surface of $\mathrm{C}_{2} \mathrm{~B}$, we prepared ${ }^{13} \mathrm{C}$-labeled $\mathrm{C}_{2} \mathrm{AB}$ mutants in which two basic residues were replaced with glutamates and then analyzed binding in $125 \mathrm{mM} \mathrm{KSCN}$ to minimize interactions that contribute to aggregation, as we used for the PCS measurements. Importantly, three double mutations that replaced basic residues in the concave side of $\mathrm{C}_{2} \mathrm{~B}$ (K313E K325E, R322E K325E and K325E K327E) strongly impaired binding to the SNARE complex (Fig. 6b), whereas binding was not 
Figure 6 Mutagenesis verifies the $\mathrm{C}_{2} \mathrm{~B}-\mathrm{SNARE}$ complex binding mode. (a) $1 \mathrm{D}^{13} \mathrm{C}$-edited ${ }^{1} \mathrm{H}$ NMR spectra of $10 \mu \mathrm{M}{ }^{13} \mathrm{C}$-labeled wild-type (WT) or R322E K325E mutant $\mathrm{C}_{2} \mathrm{AB}$ with or without $15 \mu \mathrm{M}$ SNARE complex (SC). (b,c) Normalized SMR intensities of $10 \mu \mathrm{M}$ WT or mutant ${ }^{13} \mathrm{C}-\mathrm{C}_{2} \mathrm{AB}$ as a function of SNARE-complex concentration (b) or of $10 \mu \mathrm{M}$ WT ${ }^{13} \mathrm{C}-\mathrm{C}_{2} \mathrm{AB}$ as a function of WT or mutant SNARE-complex concentration (c) in $1 \mathrm{mM}$ $\mathrm{CaCl}_{2}$ and $125 \mathrm{mM} \mathrm{KSCN}$. Data were fitted to a single-site binding model ${ }^{34}$. (d) Binding of WT and mutant $C_{2} A B$ to dansyl-labeled liposomes as a function of $\mathrm{Ca}^{2+}$ concentration, fitted with Hill equations. (e) Apparent $\mathrm{Ca}^{2+} K_{\mathrm{d}}$ values of WT and mutant $\mathrm{C}_{2} \mathrm{AB}$ in $\mathrm{Ca}^{2+}$-dependent phospholipid binding experiments. (f) $S M R$ intensities of $3 \mu \mathrm{M}$ WT or mutant ${ }^{13} \mathrm{C}-\mathrm{C}_{2} \mathrm{AB}$ upon addition of $1 \% \mathrm{PIP}_{2}$-containing liposomes (1 mM total lipid), normalized by the intensities without liposomes. (g) Normalized SMR intensities of $10 \mu \mathrm{M}$ WT or mutant ${ }^{13} \mathrm{C}-\mathrm{C}_{2} \mathrm{AB}$ as a function of SNARE-complex concentration in the absence of $\mathrm{Ca}^{2+}$ and $\mathrm{KSCN}$. (h) 1D ${ }^{13} \mathrm{C}$-edited ${ }^{1} \mathrm{H}$ NMR spectra of $15 \mu \mathrm{M}$ SNARE complex (containing ${ }^{15} \mathrm{~N}-{ }^{2} \mathrm{H}-\mathrm{ILV}-{ }^{13} \mathrm{CH}_{3}$-labeled syntaxin-1) in the absence of $\mathrm{Ca}^{2+}$ without or with $1 \% \mathrm{PIP}_{2}$-containing liposomes

(3 mM total lipid) and/or $15 \mu \mathrm{M}$ WT $\mathrm{C}_{2} \mathrm{AB}$.

(i) Normalized SMR intensities of $15 \mu \mathrm{M}$ SNARE complex in the absence of $\mathrm{Ca}^{2+}$ and presence of $15 \mu \mathrm{M}$ WT $\mathrm{C}_{2} \mathrm{AB}$ plus different concentrations of $1 \% \mathrm{PIP}_{2}$-containing liposomes. Averages from two experiments, which were sufficient to support the conclusions drawn from these data, are shown in $\mathbf{e}, \mathbf{f}$ and $\mathbf{i}$ (Online Methods); error bars, s.d.

affected by control double mutations in basic residues (K297E R388E and K354E R388E;

Fig. 1a). Moreover, double mutation of the two residues located in the polybasic region but not in the concave side of $\mathrm{C}_{2} \mathrm{~B}$ (K324E K326E) impaired binding but to a much lesser extent than the mutations in the concave side (Fig. 6b). The apparent $K_{d}$ values derived from these data (Supplementary Fig. 6c) give a semiquantitative idea of the effects of the mutations and clearly confirm the conclusion that the concave side of $\mathrm{C}_{2} \mathrm{~B}$ is primarily responsible for binding to the SNARE complex under these conditions. Furthermore, we performed titrations of wild-type (WT) ${ }^{13} \mathrm{C}-\mathrm{C}_{2} \mathrm{AB}$ with SNARE complexes containing double mutations (SNAP-25 E52K E55K and syntaxin-1 E228K D231K) in residues of the polyacidic regions of syntaxin-1 and SNAP-25 that form the primary binding sites for $\mathrm{C}_{2} \mathrm{~B}$, according to the PCS data. These mutations markedly impaired binding, whereas a control mutation in another acidic region (SNAP-25 E24K E27K) had no effect (Fig. 6c and Supplementary Fig. 6d). These results provide strong support for the binding mode derived from the PCS data.

Because $\mathrm{Ca}^{2+}$-dependent phospholipid binding is crucial for Syt1 function $^{6}$, we analyzed the effects of selected double mutations in $\mathrm{C}_{2} \mathrm{AB}$ on this activity with a fluorescence resonance energy transfer (FRET) assay (Fig. 6d). The relative effects of the mutations on the apparent $K_{\mathrm{d}}$ values observed in these titrations were distinct from those caused upon SNARE-complex binding: R322E K325E had the most marked effect on $\mathrm{Ca}^{2+}$-dependent phospholipid binding; K313E K325E and K324E K326E had similar moderate effects; and the control K354E R388E mutation had the smallest effect (Fig. 6e). We also analyzed the b

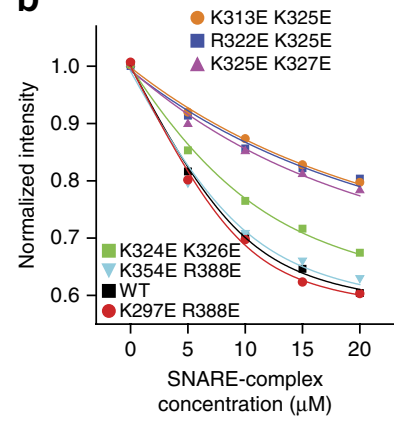

e 60

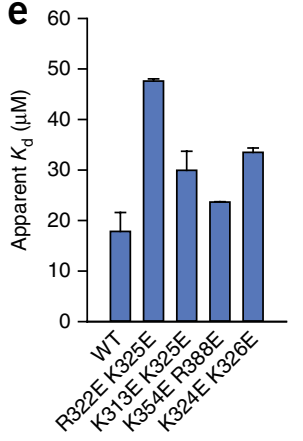

C

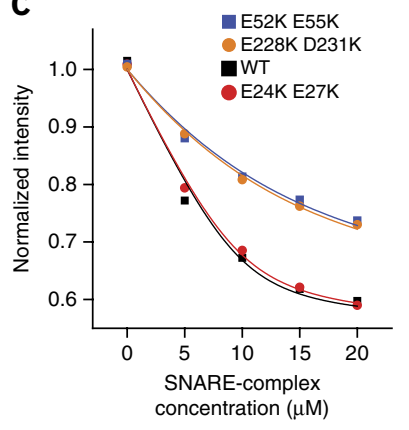

f

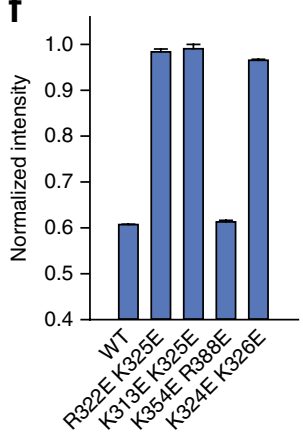

i h

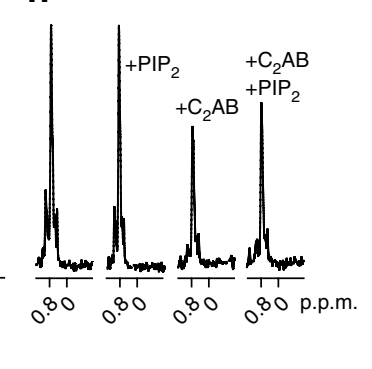

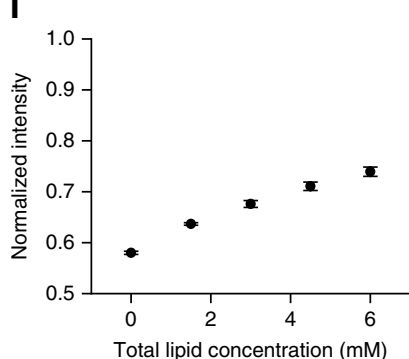

effects of these mutations on $\mathrm{Ca}^{2+}$-independent binding of $\mathrm{C}_{2} \mathrm{AB}$ to phosphatidylinositol 4,5-bisphosphate $\left(\mathrm{PIP}_{2}\right)$, which has been proposed to direct Syt 1 to the plasma membrane ${ }^{47}$. Because binding of $\mathrm{C}_{2} \mathrm{AB}$ to $\mathrm{PIP}_{2}$-containing liposomes is weak and not easily detectable (for example, by FRET assays ${ }^{48}$ ), we again used $1 \mathrm{D}^{13} \mathrm{C}$-edited ${ }^{1} \mathrm{H}$ NMR spectra of ${ }^{13} \mathrm{C}-\mathrm{C}_{2} \mathrm{AB}$, which are expected to exhibit marked decreases in SMR intensity even for small percentages of binding. Liposomes containing $1 \% \mathrm{PIP}_{2}$ (1 mM total lipid) caused a $40 \%$ decrease in the SMR intensities of WT $\mathrm{C}_{2} \mathrm{AB}$ (Fig. $6 \mathbf{f}$ ), thus confirming that a fraction of $\mathrm{C}_{2} \mathrm{AB}$ binds to the liposomes, but this binding is weak. The double mutations in the polybasic region, but not the control K354E R388E mutation, abolished binding regardless of whether the side chains were in the concave side of $\mathrm{C}_{2} \mathrm{~B}$ (Fig. $6 \mathrm{f}$ ). These data confirm that the $\mathrm{C}_{2} \mathrm{~B}$ polybasic region mediates $\mathrm{Ca}^{2+}$-independent $\mathrm{PIP}_{2}$ binding to Syt1 (ref. 47) and suggest that mutations in this region disrupt $\mathrm{PIP}_{2}$ binding more indiscriminately than they disrupt SNARE-complex binding.

A question that arises is whether PIP $_{2}$ could prevent binding of Syt 1 to the SNARE complex before $\mathrm{Ca}^{2+}$ influx. To address this question, we first examined the effects of the $\mathrm{C}_{2} \mathrm{~B}$ double mutations on $\mathrm{Ca}^{2+}$ independent binding of $\mathrm{C}_{2} \mathrm{AB}$ to the SNARE complex. We used $1 \mathrm{D}$ ${ }^{13} \mathrm{C}$-edited ${ }^{1} \mathrm{H}$ NMR spectra and $\mathrm{KSCN}$-free buffer because $\mathrm{C}_{2} \mathrm{AB}-$ SNARE complexes aggregate less in the absence of $\mathrm{Ca}^{2+}$, and these conditions facilitate the observation of $\mathrm{Ca}^{2+}$-independent binding, which is weaker than $\mathrm{Ca}^{2+}$-dependent binding ${ }^{34}$. The relative effects of the three double mutations in the $\mathrm{C}_{2} \mathrm{~B}$ polybasic region (Fig. $\mathbf{6 g}$ ) 
Figure 7 Disruption of Syt 1 function correlates with impairment of Syt1-SNARE-complex binding. (a,d) Sample traces of evoked IPSCs (eIPSCs) observed in cultured Syt1-KO neurons without or with lentiviral expression of wild-type (WT) or mutant Syt1, as indicated. Stimulus onset is indicated by the tick mark. $(\mathbf{b}, \mathbf{c}, \mathbf{e}, \mathbf{f})$ Summary graphs of the elPSC amplitudes and charge transfers in the rescue experiments with WT and mutant Syt1. $(\mathrm{g}, \mathrm{j})$ Sample traces of spontaneous release in excitatory (g) or inhibitory (j) neurons from Syt1-KO mice without or with lentiviral expression of WT Syt1 or selected Syt1 double mutants, as indicated. (h, $\mathbf{i}, \mathbf{k}, \mathbf{l})$ Summary graphs of spontaneous miniature excitatory postsynaptic current (mEPSC) $(\mathbf{h}, \mathbf{i})$ and miniature IPSC (mIPSC) (k,I) frequencies and amplitudes. All data are means \pm s.e.m.; numbers in bars indicate number of neurons/ independent cultures analyzed. Statistical significance was assessed by one-way ANOVA $\left({ }^{* *} P<0.001 ; \mathrm{NS}\right.$, not significant).

paralleled those observed in the presence of $\mathrm{Ca}^{2+}$ (Fig. 6b), thus supporting the notion that the primary $\mathrm{Ca}^{2+}$-independent and $\mathrm{Ca}^{2+}$-dependent $\mathrm{C}_{2} \mathrm{AB}-\mathrm{SNARE}$-complex binding modes are similar under these conditions. To test whether $\mathrm{PIP}_{2}$ binding to Syt 1 precludes binding to the SNARE complex, we used a competition assay monitoring $1 \mathrm{D}$ ${ }^{13} \mathrm{C}$-edited ${ }^{1} \mathrm{H}$ NMR spectra of SNARE complex containing ${ }^{15} \mathrm{~N}-{ }^{2} \mathrm{H}-\mathrm{ILV}-{ }^{13} \mathrm{CH}_{3}$-labeled syntaxin-1. Liposomes containing $1 \% \mathrm{PIP}_{2}$ ( $3 \mathrm{mM}$ total lipid) had no effect on the SMR intensity of $15 \mu \mathrm{M}$ SNARE complex (Fig. 6h), thus showing that the SNARE complex does not bind to the liposomes, whereas $15 \mu \mathrm{M}$ WT $\mathrm{C}_{2} \mathrm{AB}$ caused a marked decrease in the SMR intensity (Fig. 6h), consistently with the expectation, based on the titrations (Fig. 6g) and previous data ${ }^{46}$, that binding is close to quantitative. Addition of $\mathrm{PIP}_{2}$ containing liposomes to $15 \mu \mathrm{M} \mathrm{WT} \mathrm{C}_{2} \mathrm{AB}$ and $15 \mu \mathrm{M}$ SNARE complex yielded a modest recovery of the SMR intensity, thus reflecting partial release of $\mathrm{C}_{2} \mathrm{AB}$ from the SNARE complex (Fig. 6h); however, only $38 \%$ of the signal was recovered even with a large excess of $\mathrm{PIP}_{2}$ (6 mM total lipids; Fig. 6i). Hence, $\mathrm{PIP}_{2}$ hinders but does not abrogate $\mathrm{Ca}^{2+}$-independent binding of $\mathrm{C}_{2} \mathrm{AB}$ to the SNARE complex.

\section{Correlation of Syt1-SNARE-complex binding with Syt1 function}

To investigate the functional relevance of the Syt1-SNARE-complex binding mode described above, we performed electrophysiological rescue experiments. As previously described ${ }^{49}$, lentiviral expression of WT Syt1 rescued evoked release in Syt1-knockout (KO) neurons (Fig. 7a-c). Three single Syt1 mutants with substitutions in residues from the polybasic region (K313E, R322E and K326E) rescued evoked release almost as efficiently as WT Syt1 (Fig. 7a-c), in correlation with the finding that such mutations do not markedly impair $\mathrm{C}_{2} \mathrm{AB}-$ SNARE-complex binding (Supplementary Fig. 6a,b). Importantly

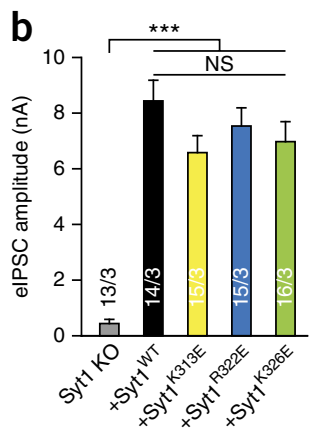

C
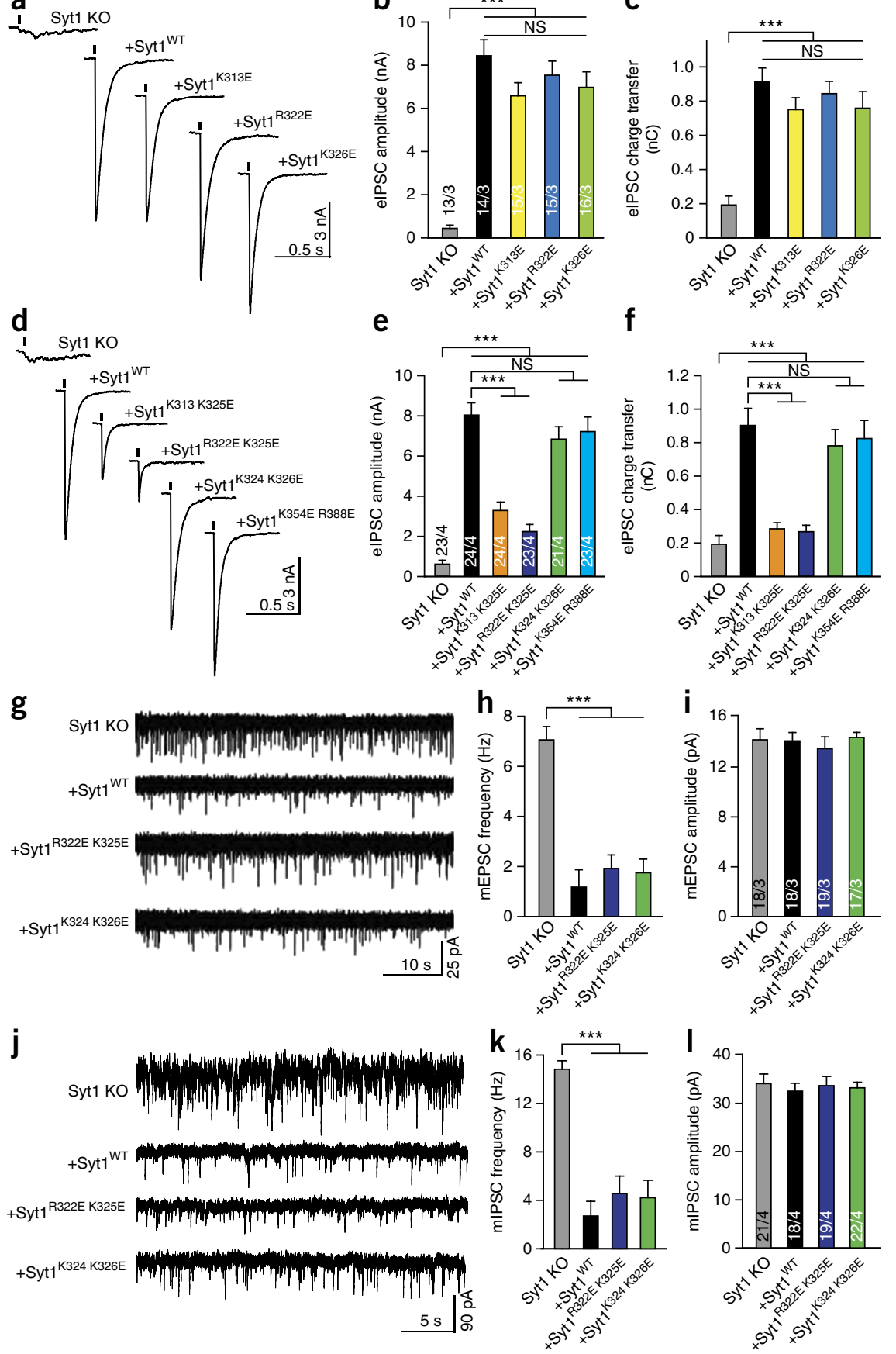
Figure 8 Disruption of Syt1-SNARE-complex binding correlates with impairment of the $\mathrm{Ca}^{2+}$-triggered step of release. (a) Sample traces of evoked IPSCs observed in Syt1-KO cultured neurons rescued with wild-type (WT) or double-mutant Syt 1 cDNAs as a function of extracellular $\mathrm{Ca}^{2+}$ concentration. The same neuron was recorded at the indicated $\mathrm{Ca}^{2+}$ concentrations. (b,c,f,g) Peak eIPSC amplitude $(\mathbf{b}, \mathbf{c})$ or total charge transfer in a single action potential $(\mathbf{f}, \mathbf{g})$, as a function of extracellular $\mathrm{Ca}^{2+}$ concentration in the rescue experiments with WT or double-mutant Syt1. Data were fit with Hill equations to determine $\mathrm{EC}_{50} \mathrm{~s}$ and Hill coefficients.

The data are presented in absolute values $(\mathbf{b}, \mathbf{f})$ or are normalized to the limiting values at infinite extracellular $\mathrm{Ca}^{2+}$ derived from the fits $(\mathbf{c}, \mathbf{g})$. In the top of $\mathbf{b}$, numbers indicate number of neurons/independent cultures analyzed. (d,e, h,i) Summary graphs of the $E_{50} \mathrm{~S}$ and Hill coefficients calculated from the data in $\mathbf{b}$ and $\mathbf{f}$. (j) Coimmunoprecipitation with syntaxin-1 (Synt IP), performed in Syt1-KO neurons rescued with WT or R322E K325E mutant Syt1 by incubation with a polyclonal antibody to syntaxin-1 and western blot analysis with monoclonal antibodies to Syt1 or Syb2. rSyt1, rat Syt1; pre, preimmune sera. (k) Quantitative analysis of

coimmunoprecipitation of WT and R322E K325E Syt1s with antibody to syntaxin-1. The amount of signal for each condition is quantified as a percentage of the input levels in that condition. All data are means \pm s.e.m. Statistical significance $(\mathbf{d}, \mathbf{e}, \mathbf{h}, \mathbf{i})$ was assessed by one-way ANOVA (** $P<0.01)$
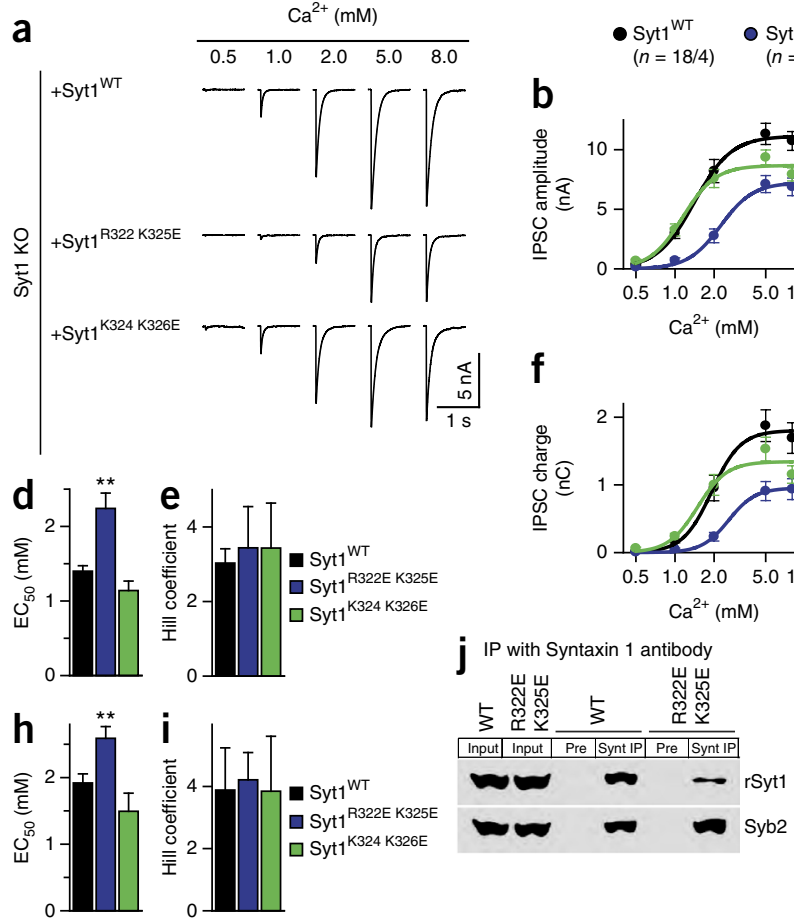

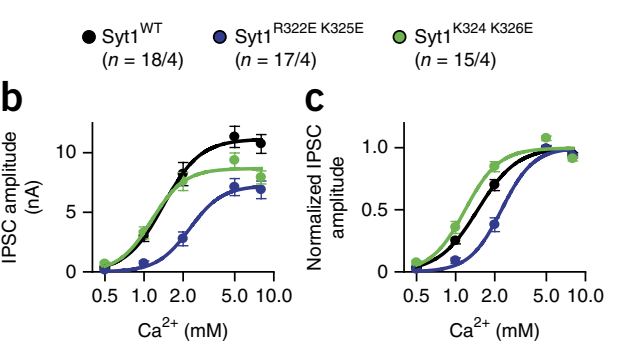

$\mathbf{f}$
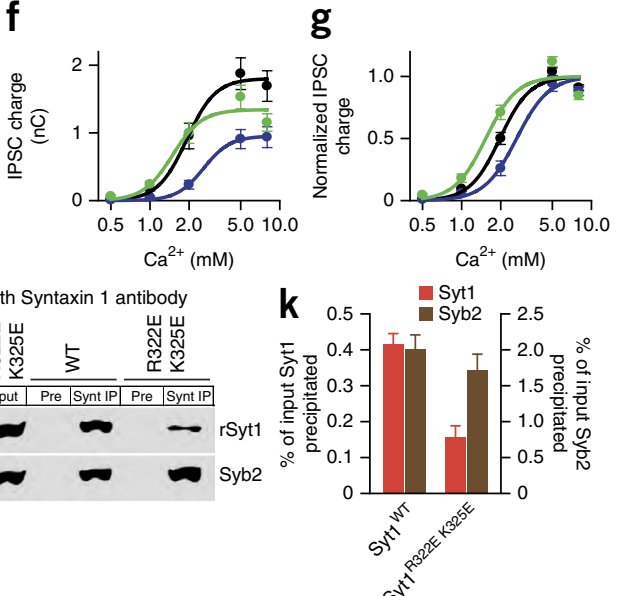

As described earlier ${ }^{50}$, overexpression of WT Syt1 suppressed the large increase in spontaneous release observed in excitatory and inhibitory Syt1-KO neurons without altering the amplitudes of individual miniature excitatory and inhibitory postsynaptic currents (Fig. 7g-1). Overexpression of all double mutants led to similar suppression of the increased miniature currents, thus suggesting that Syt1-SNARE-complex interactions are not critical for clamping the secondary $\mathrm{Ca}^{2+}$ sensor that mediates the increase in spontaneous release in Syt1-KO neurons.

We furthermore measured the dependence of evoked release on extracellular $\mathrm{Ca}^{2+}$ in Syt1-KO neurons rescued with WT Syt1 or the two double mutants (Fig. 8). Analysis of the $\mathrm{Ca}^{2+}$ dependence of both amplitude and charge transfer of inhibitory postsynaptic currents (IPSCs) further illustrated the strong impairment of evoked release caused by the R322E K325E mutation and showed that the half-maximal effective concentration $\left(\mathrm{EC}_{50}\right)$ for extracellular $\mathrm{Ca}^{2+}$ is significantly increased for rescue with this mutant compared to rescue with WT Syt1 (Fig. 8d,h). In contrast, rescue with the K324E K326E mutant did not cause a significant shift in the $\mathrm{EC}_{50}$, as compared to the WT rescue, but did result in a slight decrease in release at higher extracellular $\mathrm{Ca}^{2+}$ concentrations. These results suggest that Syt1-SNARE-complex interactions may play a key part in $\mathrm{Ca}^{2+}$ triggering of release. To examine whether the R322E K325E mutation impairs Syt1-SNARE interactions in neurons, we performed coimmunoprecipitations with an antibody to syntaxin-1. The R322E K325E mutation caused a $62 \%$ decrease in the amount of Syt 1 that coimmunoprecipitated with syntaxin-1 without significantly affecting the coimmunoprecipitation of synaptobrevin used as a control (Fig. 8j,k). This result further supports the notion that the partial impairment of neurotransmitter release caused by the R322E K325E mutation arises because of partial disruption of Syt1-SNARE interactions.

\section{DISCUSSION}

Syt1-SNARE-complex interactions are probably key for coupling $\mathrm{Ca}^{2+}$ sensing to membrane fusion during neurotransmitter release. This work illustrates the difficulties involved in studying such interactions and shows how a PCS-based approach allowed us to define a dynamic structure that represents the preferred Syt1-SNARE-complex binding mode under the specific conditions of our experiments. Our results need to be interpreted with caution because of the potential existence of other binding modes that may be occluded under our conditions. However, the relevance of our PCS-derived structure is supported by biochemical and functional data, and its dynamic nature may be an intrinsic feature that enables the fast speed of neurotransmitter release, concordantly with the increasing realization that dynamics can be key for biological function ${ }^{51}$. The dynamic Syt1-SNARE-complex structure suggests a possible mechanism for how Syt 1 relieves the inhibition caused by CpxI and supports a speculative model whereby Syt 1 brings the synaptic vesicle and plasma membrane together upon $\mathrm{Ca}^{2+}$ influx, cooperating with the SNAREs in membrane fusion (Supplementary Fig. 8).

Our analysis shows that no single structure of the $\mathrm{C}_{2} \mathrm{~B}-\mathrm{SNARE}$ complex can fit all the PCS data, which hinders application of standard computational tools to interpret these data. The manual procedure used to match the $\mathrm{C}_{2} \mathrm{~B}$ PCS patterns with the lobes of the tensors determined with the SNARE-complex PCSs does yield an approximate but unambiguous definition of the preferred location and orientation of $\mathrm{C}_{2} \mathrm{~B}$ with respect to the SNARE complex under our conditions, thus leading to a clear picture of the binding mode that readily explains its dynamic nature. Although the data used for our structural analysis were acquired in $125 \mathrm{mM} \mathrm{KSCN}$ with R398Q R399Q mutant $\mathrm{C}_{2} \mathrm{~B}$, the measured PCSs parallel those observed with WT $\mathrm{C}_{2} \mathrm{~B}$ and $\mathrm{C}_{2} \mathrm{AB}$ (Supplementary Fig. 2a,b,d,e), and the validity of the derived binding mode is supported by in vitro binding assays (Fig. 6b,c), physiological data (Figs. 7 and 8) and coimmunoprecipitation 
experiments (Fig. 8j,k). Moreover, this binding mode is consistent with results from many previous studies. Syt1 binding was previously ascribed to syntaxin-1 (example in ref. 25) or SNAP-25 (example in ref. 27); our data show that both SNAREs contribute to binding of the SNARE complex to Syt1. Our results also correlate with those from studies that mapped the Syt1-binding region to residues D51, E52 and E55 of SNAP-25 (examples in refs. 30,52), and they show that the adjacent acidic residues of syntaxin-1 also contribute to binding. Our data also agree with results that mapped the SNARE binding site to the polybasic $\beta$-strand of the $\mathrm{C}_{2} \mathrm{~B}$ domain (refs. $30-32,34,53,54$ ), but they make key distinctions between residues located at the concave side of the $\beta$-sandwich that are crucial for SNARE-complex binding and residues not located in the concave side that have less prominent roles in binding. Moreover, our results correlate very well with EPR data on $\mathrm{C}_{2} \mathrm{AB}-\mathrm{SNARE}$-complex binding ${ }^{32}$.

The present dynamic structure of the Syt1-SNARE complex is consistent with an attractive model for how Syt1 relieves the inhibition of neurotransmitter release caused by CpxI (refs. 14-16) and cooperates with the SNAREs in membrane fusion (Supplementary Fig. 8). The model postulates that, before $\mathrm{Ca}^{2+}$ influx, $\mathrm{C}_{2} \mathrm{~B}$ binds to partially assembled SNARE complexes (Supplementary Fig. 8a) via similar interactions to those defined here; this is supported by our $\mathrm{Ca}^{2+}$-independent $\mathrm{C}_{2} \mathrm{AB}-\mathrm{SNARE}$-complex binding assays (Fig. 6g) and by studies suggesting that $\mathrm{Ca}^{2+}$-independent binding of Syt1 to syntaxin-1-SNAP-25 heterodimers involves the same surfaces ${ }^{52,53}$. Notably, although the interaction is weaker in the absence of $\mathrm{Ca}^{2+}$ (Supplementary Note 2), it should be strongly enhanced by colocalization and could cooperate with binding of Arg398 and Arg399 to the plasma membrane. In this putative primed state, CpxI could be simultaneously bound to the SNARE complex because the CpxI- and Syt1-binding sites are distinct, and the negatively charged accessory helix of CpxI would inhibit release because of repulsion with the vesicle membrane $e^{22}$ (Fig. 5e and Supplementary Fig. 8a). According to our structure, in this primed state, the $\mathrm{C}_{2} \mathrm{~B}$ domain would be ideally positioned to quickly bind simultaneously to the vesicle membrane via its $\mathrm{Ca}^{2+}$-binding loops and to the plasma membrane through the bottom side upon $\mathrm{Ca}^{2+}$ influx (Supplementary Fig. 8b). Our model proposes that this action forces melting of the CpxI accessory helix (Supplementary Fig. 8b and Supplementary Note 3) and occurs concomitantly with full SNARE-complex zippering, thus leading to membrane fusion and neurotransmitter release. This model is based in part on the observation that simultaneous binding of Syt 1 to two membranes brings them within $4 \mathrm{~nm}$ of each other (refs. 11,55), and it accounts for the critical role of Arg398 and Arg399 in neurotransmitter release ${ }^{12}$. The proposed action of Syt 1 would probably require some reorientation of $\mathrm{C}_{2} \mathrm{~B}$ with respect to the SNARE complex for optimal efficiency. The dynamic nature of the $\mathrm{C}_{2} \mathrm{~B}-\mathrm{SNARE}$-complex binding mode may be a key feature facilitating such rearrangement.

Although the model in Supplementary Figure 8 potentially explains a large amount of experimental evidence, further research will be required to test its relevance and to address several unresolved issues. First, the change in $\mathrm{Ca}^{2+}$-dependent phospholipid binding to the R322E K325E mutant (Fig. 6d,e) correlates with the shift in the $\mathrm{Ca}^{2+}$ dependence of release caused by this mutant (Fig. 8). This shift can also be explained by the disruption of SNARE-complex binding caused by the R322E K325E mutation, given the synergy between $\mathrm{Ca}^{2+}$ and SNARE-complex binding to Syt1 (Supplementary Note 2), and the correlation between disruption of $\mathrm{Ca}^{2+}$-dependent phospholipid binding and impairment of Syt1 function is only partial (Figs. 6d,e and 7). These observations argue against the notion that the functional effects caused by the double mutants arise from alteration of phospholipid binding, but the finding that the mutations can affect binding both to the SNARE complex and to phospholipids underlines the limitations of studying these interactions separately because they are likely to influence each other (Supplementary Note 4). Second, there are additional binding modes between Syt 1 and the SNAREs ${ }^{29,31-34}$ that, although less populated under our specific conditions than the mode defined here, could be critical for function (Supplementary Note 5). Third, although the effects of the $\mathrm{C}_{2} \mathrm{~B}$ double mutations on SNARE-complex binding, as compared to their effects on PIP $_{2}$ binding, correlate better with disruption of Syt 1 function (Figs. 6b,f and 7), and our competition assays (Fig. 6i) suggest that the presence of $\mathrm{PIP}_{2}$ in the plasma membrane should not abrogate binding of Syt 1 to the SNARE complex, the interplay between interactions of Syt 1 with PIP $_{2}$ and the SNARE complex needs to be further investigated (Supplementary Note 6). Fourth, although our model is consistent with the importance of $\mathrm{Ca}^{2+}$-binding to the Syt $1 \mathrm{C}_{2} \mathrm{~B}$ domain ${ }^{10}$, normal release also depends on the $\mathrm{C}_{2} \mathrm{~A}$ domain ${ }^{6,56,57}$. In our $\mathrm{C}_{2} \mathrm{~B}$-SNARE-complex structure, $\mathrm{C}_{2} \mathrm{~A}$ emerges at the $\mathrm{N}$ terminus of $\mathrm{C}_{2} \mathrm{~B}$, on the opposite side of the SNARE-binding region (Fig. 5e). This positioning would allow $\mathrm{C}_{2} \mathrm{~A}$ to bind to the vesicle and/or plasma membranes and to cooperate with the action of $\mathrm{C}_{2} \mathrm{~B}$ in triggering release. However, there is some evidence that $\mathrm{C}_{2} \mathrm{~A}-\mathrm{SNARE}$ interactions are important for release ${ }^{13,58}$, and there are weak $\mathrm{C}_{2} \mathrm{~A}-\mathrm{SNARE}$ complex interactions that contribute to aggregation of $\mathrm{C}_{2} \mathrm{AB}-\mathrm{SNARE}$ complexes in solution ${ }^{34}$ but could be functionally important.

Challenging structural studies of Syt1-SNARE-complex interactions on membranes, or ideally between two membranes, will probably be required to resolve these and other issues. Despite all these concerns, the dynamic structure of the Syt1-SNARE complex described here provides a framework for rationalization of the available data and will serve as a guide for future research in this field.

\section{METHODS}

Methods and any associated references are available in the online version of the paper.

Accession codes. Coordinates of five energy-minimized structures that illustrate the dynamic Syt $1 \mathrm{C}_{2} \mathrm{~B}$ domain-SNARE-complex binding mode have been deposited in the Protein Data Bank under accession code $2 \mathrm{~N} 1 \mathrm{~T}$. The ensemble includes the 166-MD model and four of the structures from the chemical shift-restrained MD simulations that contribute to the optimized population-weighted PCS data of Figure 4e,f. The five conformers deposited must be considered to be just a few of the many conformers that form the dynamic ensemble existing under our conditions, but they illustrate the types of interactions between the Syt $1 \mathrm{C}_{2} \mathrm{~B}$ domain and the SNARE complex that mediate this dynamic binding mode.

Note: Any Supplementary Information and Source Data files are available in the online version of the paper.

\section{ACKNOWLEDGMENTS}

We thank L. Kay for fruitful discussions and F. Peters (Max Planck Institute for Biophysical Chemistry) for generously providing a sample for Cys-Ph-TAHAlabeling. The DD2 console of one of the Agilent 600-MHz NMR spectrometers used for the research presented here was purchased with shared instrumentation grant S10RR026461 from the US National Institutes of Health (to M.K. Rosen; University of Texas Southwestern Medical Center). The authors acknowledge the Texas Advanced Computing Center at the University of Texas at Austin for providing high-performance computer resources that have contributed to the research results reported within this paper. This work was supported by Welch Foundation grant I-1304 (to J.R.), Australian Research Council (ARC) Discovery Grant DP150100383 (to B.G.), ARC Future Fellowship FT130100838 (to B.G.), Swiss National Science Foundation grant 200021_130263 (to D.H.) and US National Institutes of Health grants K99NS087086 (to T.B.) and NS040944 (to J.R.). 


\section{AUTHOR CONTRIBUTIONS}

K.D.B., T.B., J.D.S., A.Z., P.Z., N.B., J.X., A.B.S. and E.A.P. performed experiments and analyzed data. A.C., C.C., J.L. and R.V. performed computational analyses. D.H., A.M.J.J.B., D.R.T., M.V., B.G. and T.C.S. designed experiments and analyzed data. J.R. analyzed data and wrote the manuscript with input from all coauthors.

\section{COMPETING FINANCIAL INTERESTS}

The authors declare no competing financial interests.

Reprints and permissions information is available online at http://www.nature.com/ reprints/index.html.

1. Brunger, A.T., Weninger, K., Bowen, M. \& Chu, S. Single-molecule studies of the neuronal SNARE fusion machinery. Annu. Rev. Biochem. 78, 903-928 (2009).

2. Rizo, J. \& Sudhof, T.C. The membrane fusion enigma: SNAREs, Sec1/Munc18 proteins, and their accomplices-guilty as charged? Annu. Rev. Cell Dev. Biol. 28, 279-308 (2012)

3. Poirier, M.A. et al. The synaptic SNARE complex is a parallel four-stranded helical bundle. Nat. Struct. Biol. 5, 765-769 (1998).

4. Sutton, R.B., Fasshauer, D., Jahn, R. \& Brunger, A.T. Crystal structure of a SNARE complex involved in synaptic exocytosis at 2.4 A resolution. Nature $395,347-353$ (1998).

5. Hanson, P.I., Roth, R., Morisaki, H., Jahn, R. \& Heuser, J.E. Structure and conformational changes in NSF and its membrane receptor complexes visualized by quick-freeze/deep-etch electron microscopy. Cell 90, 523-535 (1997).

6. Fernández-Chacón, R. et al. Synaptotagmin I functions as a calcium regulator of release probability. Nature 410, 41-49 (2001).

7. Sutton, R.B., Davletov, B.A., Berghuis, A.M., Sudhof, T.C. \& Sprang, S.R. Structure of the first $\mathrm{C} 2$ domain of synaptotagmin I: a novel $\mathrm{Ca}^{2+} /$ phospholipid-binding fold. Cell 80, 929-938 (1995).

8. Ubach, J., Zhang, X., Shao, X., Sudhof, T.C. \& Rizo, J. $\mathrm{Ca}^{2+}$ binding to synaptotagmin: how many $\mathrm{Ca}^{2+}$ ions bind to the tip of a C2-domain? EMBO J. 17, 3921-3930 (1998).

9. Fernandez, I. et al. Three-dimensional structure of the synaptotagmin $1 \mathrm{C}_{2} \mathrm{~B}$-domain: synaptotagmin 1 as a phospholipid binding machine. Neuron 32, 1057-1069 (2001).

10. Mackler, J.M. \& Reist, N.E. Mutations in the second C2 domain of synaptotagmin disrupt synaptic transmission at Drosophila neuromuscular junctions. J. Comp. Neurol. 436, 4-16 (2001).

11. Araç, D. et al. Close membrane-membrane proximity induced by $\mathrm{Ca}^{2+}$-dependent multivalent binding of synaptotagmin-1 to phospholipids. Nat. Struct. Mol. Biol. 13, 209-217 (2006)

12. Xue, M., Ma, C., Craig, T.K., Rosenmund, C. \& Rizo, J. The Janus-faced nature of the $\mathrm{C}_{2} \mathrm{~B}$ domain is fundamental for synaptotagmin-1 function. Nat. Struct. Mol. Biol. 15, 1160-1168 (2008).

13. Pang, Z.P., Shin, O.H., Meyer, A.C., Rosenmund, C. \& Sudhof, T.C. A gain-offunction mutation in synaptotagmin-1 reveals a critical role of $\mathrm{Ca}^{2+}$-dependent soluble $\mathrm{N}$-ethylmaleimide-sensitive factor attachment protein receptor complex binding in synaptic exocytosis. J. Neurosci. 26, 12556-12565 (2006).

14. Tang, J. et al. A complexin/synaptotagmin 1 switch controls fast synaptic vesicle exocytosis. Cell 126, 1175-1187 (2006)

15. Schaub, J.R., Lu, X., Doneske, B., Shin, Y.K. \& McNew, J.A. Hemifusion arrest by complexin is relieved by $\mathrm{Ca}^{2+}{ }^{2+}$ synaptotagmin I. Nat. Struct. Mol. Biol. 13, 748-750 (2006).

16. Giraudo, C.G., Eng, W.S., Melia, T.J. \& Rothman, J.E. A clamping mechanism involved in SNARE-dependent exocytosis. Science 313, 676-680 (2006).

17. Reim, K. et al. Complexins regulate a late step in $\mathrm{Ca}^{2+}$-dependent neurotransmitter release. Cell 104, 71-81 (2001).

18. Huntwork, S. \& Littleton, J.T. A complexin fusion clamp regulates spontaneous neurotransmitter release and synaptic growth. Nat. Neurosci. 10, 1235-1237 (2007).

19. Maximov, A., Tang, J., Yang, X., Pang, Z.P. \& Sudhof, T.C. Complexin controls the force transfer from SNARE complexes to membranes in fusion. Science $\mathbf{3 2 3}$, 516-521 (2009).

20. Chen, X. et al. Three-dimensional structure of the complexin/SNARE complex. Neuron 33, 397-409 (2002).

21. Xue, M. et al. Distinct domains of complexin I differentially regulate neurotransmitter release. Nat. Struct. Mol. Biol. 14, 949-958 (2007).

22. Trimbuch, T. et al. Re-examining how complexin inhibits neurotransmitter release. eLife. 3, e02391 (2014).

23. Ma, C., Su, L., Seven, A.B., Xu, Y. \& Rizo, J. Reconstitution of the vital functions of Munc18 and Munc13 in neurotransmitter release. Science 339, 421-425 (2013).

24. Rizo, J., Chen, X. \& Arac, D. Unraveling the mechanisms of synaptotagmin and SNARE function in neurotransmitter release. Trends Cell Biol. 16, 339-350 (2006).

25. Bennett, M.K., Calakos, N. \& Scheller, R.H. Syntaxin: a synaptic protein implicated in docking of synaptic vesicles at presynaptic active zones. Science 257, 255-259 (1992)

26. $\mathrm{Li}, \mathrm{C}$. et al. $\mathrm{Ca}^{2+-d e p e n d e n t ~ a n d ~-i n d e p e n d e n t ~ a c t i v i t i e s ~ o f ~ n e u r a l ~ a n d ~ n o n-n e u r a l ~}$ synaptotagmins. Nature 375, 594-599 (1995).

27. Gerona, R.R., Larsen, E.C., Kowalchyk, J.A. \& Martin, T.F. The C terminus of SNAP25 is essential for $\mathrm{Ca}^{2+}$-dependent binding of synaptotagmin to SNARE complexes. J. Biol. Chem. 275, 6328-6336 (2000).
28. Chapman, E.R., Hanson, P.I., An, S. \& Jahn, R. Ca ${ }^{2+}$ regulates the interaction between synaptotagmin and syntaxin 1. J. Biol. Chem. 270, 23667-23671 (1995).

29. Zhang, X., Kim-Miller, M.J., Fukuda, M., Kowalchyk, J.A. \& Martin, T.F. $\mathrm{Ca}^{2+}$ dependent synaptotagmin binding to SNAP-25 is essential for $\mathrm{Ca}^{2+}$-triggered exocytosis. Neuron 34, 599-611 (2002).

30. Rickman, C. et al. Conserved prefusion protein assembly in regulated exocytosis. Mol. Biol. Cell 17, 283-294 (2006).

31. Dai, H., Shen, N., Arac, D. \& Rizo, J. A quaternary SNARE-synaptotagmin-Ca2+phospholipid complex in neurotransmitter release. J. Mol. Biol. 367, 848-863 (2007).

32. Lai, A.L., Huang, H., Herrick, D.Z., Epp, N. \& Cafiso, D.S. Synaptotagmin 1 and SNAREs form a complex that is structurally heterogeneous. J. Mol. Biol. 405, 696-706 (2011).

33. Choi, U.B. et al. Single-molecule FRET-derived model of the synaptotagmin 1-SNARE fusion complex. Nat. Struct. Mol. Biol. 17, 318-324 (2010).

34. Zhou, A., Brewer, K.D. \& Rizo, J. Analysis of SNARE complex/synaptotagmin-1 interactions by one-dimensional NMR spectroscopy. Biochemistry 52, 3446-3456 (2013).

35. Xu, J., Brewer, K.D., Perez-Castillejos, R. \& Rizo, J. Subtle interplay between synaptotagmin and complexin binding to the SNARE complex. J. Mol. Biol. $\mathbf{4 2 5}$, 3461-3475 (2013)

36. Ruschak, A.M. \& Kay, L.E. Methyl groups as probes of supra-molecular structure, dynamics and function. J. Biomol. NMR 46, 75-87 (2010).

37. Otting, G. Protein NMR using paramagnetic ions. Annu. Rev. Biophys. 39, 387-405 (2010).

38. Zhang, Y. \& Cremer, P.S. Interactions between macromolecules and ions: the Hofmeister series. Curr. Opin. Chem. Biol. 10, 658-663 (2006).

39. Richens, J.L., Lunt, E.A., Sanger, D., McKenzie, G. \& O'Shea, P. Avoiding nonspecific interactions in studies of the plasma proteome: practical solutions to prevention of nonspecific interactions for label-free detection of low-abundance plasma proteins. J. Proteome Res. 8, 5103-5110 (2009).

40. de la Cruz, L. et al. Binding of low molecular weight inhibitors promotes large conformational changes in the dengue virus NS2B-NS3 protease: fold analysis by pseudocontact shifts. J. Am. Chem. Soc. 133, 19205-19215 (2011).

41. Graham, B. et al. DOTA-amide lanthanide tag for reliable generation of pseudocontact shifts in protein NMR spectra. Bioconjug. Chem. 22, 2118-2125 (2011).

42. Schmitz, C. \& Bonvin, A.M. Protein-protein HADDocking using exclusively pseudocontact shifts. J. Biomol. NMR 50, 263-266 (2011).

43. Shishmarev, D. \& Otting, G. How reliable are pseudocontact shifts induced in proteins and ligands by mobile paramagnetic metal tags? A modelling study. J. Biomol. NMR 56, 203-216 (2013).

44. Camilloni, C., Robustelli, P., De, S.A., Cavalli, A. \& Vendruscolo, M. Characterization of the conformational equilibrium between the two major substates of RNase $A$ using NMR chemical shifts. J. Am. Chem. Soc. 134, 3968-3971 (2012).

45. DeSimone, A., Montalvao, R.W. \& Vendruscolo, M. Determination of conformational equilibria in proteins using residual dipolar couplings. J. Chem. Theory Comput. 7 , 4189-4195 (2011)

46. Araç, D., Murphy, T. \& Rizo, J. Facile detection of protein-protein interactions by one-dimensional NMR spectroscopy. Biochemistry 42, 2774-2780 (2003).

47. Bai, J., Tucker, W.C. \& Chapman, E.R. PIP 2 increases the speed of response of synaptotagmin and steers its membrane-penetration activity toward the plasma membrane. Nat. Struct. Mol. Biol. 11, 36-44 (2004).

48. Radhakrishnan, A., Stein, A., Jahn, R. \& Fasshauer, D. The $\mathrm{Ca}^{2+}$ affinity of synaptotagmin 1 is markedly increased by a specific interaction of its $C 2 B$ domain with phosphatidylinositol 4,5-bisphosphate. J. Biol. Chem. 284, 25749-25760 (2009).

49. Xu, J., Mashimo, T. \& Sudhof, T.C. Synaptotagmin-1, -2, and -9: $\mathrm{Ca}^{2+}$ sensors for fast release that specify distinct presynaptic properties in subsets of neurons. Neuron 54, 567-581 (2007).

50. Xu, J., Pang, Z.P., Shin, O.H. \& Sudhof, T.C. Synaptotagmin-1 functions as a $\mathrm{Ca}^{2+}$ sensor for spontaneous release. Nat. Neurosci. 12, 759-766 (2009).

51. Mittermaier, A.K. \& Kay, L.E. Observing biological dynamics at atomic resolution using NMR. Trends Biochem. Sci. 34, 601-611 (2009).

52. Kim, J.Y. et al. Solution single-vesicle assay reveals $\mathrm{PIP}_{2}$-mediated sequential actions of synaptotagmin-1 on SNAREs. EMBO J. 31, 2144-2155 (2012).

53. Rickman, C. et al. Synaptotagmin interaction with the syntaxin/SNAP-25 dimer is mediated by an evolutionarily conserved motif and is sensitive to inositol hexakisphosphate. J. Biol. Chem. 279, 12574-12579 (2004).

54. Malsam, J. et al. Complexin arrests a pool of docked vesicles for fast $\mathrm{Ca}^{2+}$-dependent release. EMBO J. 31, 3270-3281 (2012).

55. Seven, A.B., Brewer, K.D., Shi, L., Jiang, Q.X. \& Rizo, J. Prevalent mechanism of membrane bridging by synaptotagmin-1. Proc. Natl. Acad. Sci. USA 110 E3243-E3252 (2013)

56. Shin, O.H., Xu, J., Rizo, J. \& Sudhof, T.C. Differential but convergent functions of $\mathrm{Ca}^{2+}$ binding to synaptotagmin-1 C2 domains mediate neurotransmitter release. Proc. Natl. Acad. Sci. USA 106, 16469-16474 (2009).

57. Lee, J., Guan, Z., Akbergenova, Y. \& Littleton, J.T. Genetic analysis of synaptotagmin $\mathrm{C} 2$ domain specificity in regulating spontaneous and evoked neurotransmitter release. J. Neurosci. 33, 187-200 (2013).

58. Lynch, K.L. et al. Synaptotagmin C2A loop 2 mediates $\mathrm{Ca}^{2+}$-dependent SNARE interactions essential for $\mathrm{Ca}^{2+}$-triggered vesicle exocytosis. Mol. Biol. Cell 18, 4957-4968 (2007) 


\section{ONLINE METHODS}

Protein expression and purification. The expression and purification of fragments spanning the SNARE motifs of rat synaptobrevin 2 (residues 29-93), rat syntaxin-1A (residues 191-253), and human SNAP-25 (residues 11-82 and 141-203) from a pGEX-KT vector, and rat Syt1 $\mathrm{C}_{2} \mathrm{~B}$ (residues 271-421), $\mathrm{C}_{2} \mathrm{~B}$ R398Q R399Q mutant and $\mathrm{C}_{2} \mathrm{AB}$ (residues 131-421 and 140-421) from a pGEX-KG vector were previously described ${ }^{11,20,35}$. Constructs to express singlecysteine SNARE mutants were obtained by site-directed mutagenesis with PCR and custom-designed primers. Unlabeled proteins were expressed in Escherichia coli BL21(DE3) cells in LB medium. For uniformly ${ }^{13} \mathrm{C}-{ }^{15} \mathrm{~N}$-labeled proteins, we used M9 minimal expression medium with $\left[{ }^{13} \mathrm{C}_{6}\right]$ glucose as the sole carbon source ( $3 \mathrm{~g}$ per L of culture) and ${ }^{15} \mathrm{NH}_{4} \mathrm{Cl}$ as the sole nitrogen source $(1 \mathrm{~g} / \mathrm{L})$. Perdeuterated proteins were produced with $\mathrm{M} 9$ expression medium in $99.9 \%$ $\mathrm{D}_{2} \mathrm{O}$ with $\left[{ }^{2} \mathrm{H},{ }^{12} \mathrm{C}\right]$ glucose as the sole carbon source $(3 \mathrm{~g} / \mathrm{L})$ and ${ }^{15} \mathrm{NH}_{4} \mathrm{Cl}$ as the sole nitrogen source $(1 \mathrm{~g} / \mathrm{L})$. ILVM methyl-labeling was achieved by addition of $\left[3,3-{ }^{2} \mathrm{H}_{2}\right]{ }^{13} \mathrm{C}$-methyl alpha-ketobutyric acid $(80 \mathrm{mg} / \mathrm{L}),\left[3-{ }^{2} \mathrm{H}\right]{ }^{13} \mathrm{C}$-dimethyl alpha-ketoisovaleric acid ( $80 \mathrm{mg} / \mathrm{L})$, and ${ }^{13} \mathrm{C}$-methyl methionine $(250 \mathrm{mg} / \mathrm{L})$ (Cambridge Isotope Laboratories) to the cell cultures $30 \mathrm{~min}$ before isopropyl $\beta$-D-1-thiogalactopyranoside (IPTG) induction. SNARE-complex assembly was achieved as previously described ${ }^{20}$ by mixture of the SNARE domains in equimolar ratio, except that the assembly reaction was incubated at room temperature while the mixture was rotated.

Paramagnetic labeling. The SNARE complex does not contain native cysteines. To label with paramagnetic tags, we obtained diverse single-cysteine mutants of the SNAREs (described below). For labeling of SNARE cysteine mutants with paramagnetic tags, the protein was treated with $10 \mathrm{mM} \mathrm{DTT}$, which was subsequently removed by gel-filtration chromatography on a Superdex S75 column in $25 \mathrm{mM}$ Tris-HCl, pH 7.4, $150 \mathrm{mM} \mathrm{NaCl}$. For MTSL-labeling, the protein was concentrated to $40-60 \mu \mathrm{M}$ and incubated overnight at $4{ }^{\circ} \mathrm{C}$ with a ten-fold molar excess of MTSL from a $40 \mathrm{mM}$ stock in dimethyl sulfoxide. The excess MTSL was removed by concentration-dilution in the gel-filtration buffer with a $3-\mathrm{kDa}$ molecularweight-cutoff filter before being assembled into the SNARE complex. The SNARE complex was then buffer-exchanged into $25 \mathrm{mM}$ D-Tris-DCl, pH 7.4, $125 \mathrm{mM}$ KSCN, $1 \mathrm{mM} \mathrm{CaCl}_{2}$ and $10 \% \mathrm{D}_{2} \mathrm{O}$ with a $10-\mathrm{kDa}$ molecular-weight-cutoff filter. KSCN salt was used in these and other experiments to limit nonspecific interactions and to prevent precipitation of the Syt1-SNARE complex in the presence of $\mathrm{Ca}^{2+}$. The MTSL nitroxide radical was reduced when needed by addition of $1 \mathrm{mM}$ ascorbic acid and $1 \mathrm{mM}$ sodium dithionite from $100 \mathrm{mM}$ stocks adjusted to $\mathrm{pH}$ 7.4, prepared immediately before use. For lanthanide-labeling with $\mathrm{Dy}^{3+}$-DOTA-M8 or $\mathrm{Dy}^{3+}{ }_{-} \mathrm{C} 2$, the protein after gel filtration was concentrated to $30-100 \mu \mathrm{M}$ and incubated $10 \mathrm{~min}$ at room temperature with a three-fold molar excess of the tag. After tag-labeling, the other SNAREs were added for assembly and incubated at room temperature with overnight rotation. A final concentration of $1 \mathrm{M} \mathrm{NaCl}$ was also added before mixture of the SNAREs to prevent precipitation. To minimize the amount of free isotopically labeled SNARE left after SNARE-complex assembly, nonisotopically labeled SNAREs were included in $40 \%$ excess. The SNARE complex was buffer-exchanged at $25^{\circ} \mathrm{C}$ into $25 \mathrm{mM}$ Tris- $\mathrm{HCl}, \mathrm{pH} 7.4$, $500 \mathrm{mM} \mathrm{NaCl}$ with a $10-\mathrm{kDa}$ molecular-weight-cutoff filter to remove the excess tag and SNAREs and then exchanged to $25 \mathrm{mM}$ D-Tris-DCl, $\mathrm{pH} 7.4,125 \mathrm{mM}$ KSCN, $1 \mathrm{mM} \mathrm{CaCl}_{2}, 10 \% \mathrm{D}_{2} \mathrm{O}$. The samples were reduced when needed by the addition of $0.3 \mathrm{mM}$ TCEP from a $30 \mathrm{mM}$ stock.

NMR spectroscopy. All NMR spectra were acquired at $25^{\circ} \mathrm{C}$ on Varian INOVA spectrometers operating at 600 or $800 \mathrm{MHz}$, equipped with cold probes. ${ }^{1} \mathrm{H}_{-}{ }^{13} \mathrm{C}$ HMQC and ${ }^{1} \mathrm{H}-{ }^{15} \mathrm{~N}$ HSQC-TROSY spectra were acquired under the conditions indicated in the figure legends with $10 \% \mathrm{D}_{2} \mathrm{O}$ as the solvent. Total acquisition times were 4-56 h. NMR data were processed with NMRPipe ${ }^{59}$ and analyzed with $\mathrm{NMRView}^{60}$. All data for PCS measurements were acquired in $25 \mathrm{mM}$ Tris- $\mathrm{HCl}$, $\mathrm{pH} 7.4,125 \mathrm{mM}$ KSCN, $1 \mathrm{mM} \mathrm{CaCl} 2$ and $10 \% \mathrm{D}_{2} \mathrm{O}$.

Measurement of PCSs. Analysis of PCSs was based on the assignments that are available for most of the resonances of the Syt $1 C_{2}$ domains and for the SNARE-complex backbone ${ }^{9,20,61,62}$. To measure PCSs within the SNARE complex, we acquired ${ }^{1} \mathrm{H}-{ }^{15} \mathrm{~N}$ TROSY-HSQC spectra of $60-80 \mu \mathrm{M}$ samples of SNARE complex ${ }^{2} \mathrm{H}-{ }^{15} \mathrm{~N}$-labeled at the syntaxin-1 or SNN SNARE motifs (with the $\mathrm{Dy}^{3+}{ }_{-} \mathrm{C} 2$ tag on residue 166 of SNAP-25) or at the synaptobrevin and syntaxin-1 SNARE motifs (with the $\mathrm{Dy}^{3+}{ }_{-} \mathrm{C} 2$ tag on residue 41 of SNAP-25). PCSs were calculated from the differences in the chemical shifts observed before and after removal of the $\mathrm{Dy}^{3+}-\mathrm{C} 2$ tag by reduction with $1 \mathrm{mM}$ TCEP. Because of the relatively low sensitivity of the spectra at the concentrations used, cross-peaks from NH groups within less than $\sim 18 \AA$ from the lanthanide were broadened beyond detection, and the chemical shifts of all the $\mathrm{NH}$ groups for which we could measure PCSs were not affected by introduction of a diamagnetic tag. Hence, the procedure used to measure PCSs is equivalent to using control spectra with SNARE complex tagged with a nonparamagnetic lanthanide. Errors in the measurements were estimated from the reproducibility of the chemical shifts obtained manually from contour plots of repeated spectra. The PCSs measured within the SNARE complex with $\mathrm{Dy}^{3+}{ }_{-} \mathrm{C} 2$ tag on residue 166 or 41 of SNAP-25 were used to derive the corresponding $\Delta \chi$ tensors (referred to as SC166 and SC41 tensors, respectively; Fig. 2) in Numbat ${ }^{63}$. To analyze how sensitive the tensor parameters are to small variations in the coordinates of the center, we recalculated the tensor while constraining the center to be at different random locations within $4 \AA$ of the optimal tensor center derived without constraints. Selected tensors among those obtained were used to reanalyze the fits between PCSs measured on $\mathrm{C}_{2} \mathrm{~B}$ and the calculated values (for example, Fig. $\mathbf{3 h}, \mathbf{j}$ ).

Small PCSs on many cross-peaks of the ${ }^{1} \mathrm{H}-{ }^{13} \mathrm{C}$ HMQC and ${ }^{1} \mathrm{H}-{ }^{15} \mathrm{~N}$ TROSYHSQC spectra of $30 \mu \mathrm{M} \mathrm{C} \mathrm{C}_{2} \mathrm{AB}$ were induced by $20 \mu \mathrm{M}$ SC41Dy or SC166Dy (example in Supplementary Fig. 2a,d), but higher concentrations to saturate binding more fully, and hence increase the PCSs, led to aggregation. Because the $\mathrm{C}_{2} \mathrm{~A}$ domain contributes to aggregation ${ }^{34}$, and all the stronger PCSs observed in $\mathrm{C}_{2} \mathrm{AB}$ correspond to cross-peaks from the $\mathrm{C}_{2} \mathrm{~B}$ domain, we used a Syt 1 fragment containing only the $\mathrm{C}_{2} \mathrm{~B}$ domain (residues 270-421) and were able to obtain good-quality data upon addition of $30 \mu \mathrm{M}$ SC41Dy or SC166Dy (Supplementary Fig. 2b,e). The PCSs observed for $C_{2} B$ were parallel to those observed for $C_{2} A B$ (Supplementary Fig. 2a,b,d,e), thus showing that removal of the $\mathrm{C}_{2} \mathrm{~A}$ domain did not alter the major binding mode. Very similar PCSs were also observed for $\mathrm{C}_{2} \mathrm{~B}$ with the R398Q R399Q mutation (Fig. 3a,b). We used the PCSs measured for this mutant (referred to as $\mathrm{C}_{2} \mathrm{~B}$ for simplicity) for further analysis because it yielded the best-quality data, and the PCSs are less likely to be influenced by weak binding modes that lead to aggregation. From ${ }^{1} \mathrm{H}-{ }^{15} \mathrm{~N}$ HSQC titrations (such as those shown in Fig. 1b), we estimated that binding was about $66 \%$ saturated under the conditions of the experiments. To account for the incomplete binding, all PCSs measured on $\mathrm{C}_{2} \mathrm{~B}$ were multiplied by a factor of 1.5. Because of the small size of the PCSs and because the digital resolution was much higher in the ${ }^{1} \mathrm{H}$ dimension, we measured PCSs on only ${ }^{1} \mathrm{H}$ nuclei.

Structural analysis with PCSs. We built the 166 and 41 manual models (Fig. 3e,f) in PyMOL (http://www.pymol.org/) by manual translation and rotation of the $\mathrm{C}_{2} \mathrm{~B}$ domain with respect to the SNARE complex, trying to optimally match the pattern of positive-negative PCSs with the positive-negative lobes of the SC166 and SC41 tensors, respectively, while also having the $\mathrm{C}_{2} \mathrm{~B}$ domain within van der Waals contact with the SNARE complex.

To try to obtain structures of the $\mathrm{C}_{2} \mathrm{~B}$-SNARE complex compatible with the SC166Dy PCS data computationally, we used Numbat to calculate a $\Delta \chi$ tensor from the PCSs induced on $\mathrm{C}_{2} \mathrm{~B}$ (referred to as $\mathrm{C}_{2} \mathrm{~B} 166$ tensor; Supplementary Fig. 3a) and observed a good correlation between measured PCSs and those calculated with the tensor (Supplementary Fig. 3c). However, this tensor has a considerably different shape from the tensor derived with the SNARE-complex PCSs (the SC166 tensor; comparison of Supplementary Fig. 3a,b), and $\mathrm{C}_{2} \mathrm{~B}$ does not contact the SNARE complex in the model obtained by superimposing the two tensors with PyMOL (Supplementary Fig. 3b). Moreover, the $\mathrm{C}_{2} \mathrm{~B}$ PCSs calculated with this model and the SC166 tensor have only a modest correlation with the measured PCSs (Supplementary Fig. 3d). Because of the axial symmetry of the SC166 tensor, we used PyMOL to manually rotate $\mathrm{C}_{2} \mathrm{~B}$ around the vertical axis (in the orientation of Supplementary Fig. 3b), trying to maintain a similar orientation and distance of $\mathrm{C}_{2} \mathrm{~B}$ with respect to the long axis of the SC166 tensor, as observed after the initial superposition of the $\mathrm{C}_{2} \mathrm{~B} 166$ and SC166 tensors, but placing $\mathrm{C}_{2} \mathrm{~B}$ within van der Waals contact of the SNARE complex (Supplementary Fig. 3f). The resulting model yields a similar correlation between calculated and measured $\mathrm{C}_{2} \mathrm{~B}$ PCSs (Supplementary Fig. 3e). However, in this model $\mathrm{C}_{2} \mathrm{~B}$ would clash with CpxI if Syt 1 and CpxI were bound simultaneously to the SNARE complex (Supplementary Fig. 3f). Moreover, the distance from $\mathrm{C}_{2} \mathrm{~B}$ to the center of the $\mathrm{C}_{2} \mathrm{~B} 166$ tensor (Supplementary Fig. 3a) 
is larger than the distance from $\mathrm{C}_{2} \mathrm{~B}$ to the center of the SC166 tensor in the 166-manual model (Supplementary Fig. 3g). Because fast motions of a lanthanide with respect to a molecule attenuate the observed PCSs and yield a distorted tensor with a center that is further away from the molecule than the real position of the lanthanide 43 , this analysis indicates that the PCSs induced by SC166Dy on $\mathrm{C}_{2} \mathrm{~B}$ reflect motional averaging and that the manual model constitutes a better representation of the center of the ensemble of binding modes than models derived from superposition of the $\mathrm{S} C 166$ and $\mathrm{C}_{2} \mathrm{~B} 166$ tensors.

We also attempted to obtain structures of the $\mathrm{C}_{2} \mathrm{~B}-\mathrm{SNARE}$ complex that fit the SC166Dy PCS data with HADDOCK-PCS ${ }^{42}$, but we did not obtain any structures exhibiting good correlations between observed and calculated PCSs. Similar results were obtained with the SC41Dy PCS data. To try to account for the decreases in PCSs expected to be caused by motional averaging and/or for the possibility that we overestimated the population of the major binding mode, we attempted to find structures that fit the measured PCSs multiplied by $\mathrm{x}$ factors from 2 to 7 with HADDOCK-PCS. We did not obtain structures with a good fit for the SC41Dy data. With the SC166Dy data, we did obtain structures that had consistent orientations of $\mathrm{C}_{2} \mathrm{~B}$ and had good correlations between measured and calculated PCSs, as illustrated in Supplementary Figure 4a for a representative structure obtained with an $\mathrm{x}$ factor of 5 (referred to as the 166 HADDOCK model). However, these structures exhibited a limited number of salt bridges between $\mathrm{C}_{2} \mathrm{~B}$ and the SNAREs, and the pattern of positive-negative PCSs did not match well with the positive-negative lobes of the SC166 tensor (Supplementary Fig. 4b). This overall analysis emphasizes the danger of attempting to fit dynamically averaged PCS data to single structures and shows that the large slopes in Figure 3g,h and Supplementary Figure $4 \mathbf{c}$ arise to a large extent from motions of $\mathrm{C}_{2} \mathrm{~B}$ with respect to the SNARE complex.

To aid in the analysis of PCSs, we performed unrestrained MD simulations starting from the 166 HADDOCK model (Supplementary Note 8) and generated a chemical shift-based ensemble with replica-averaged metadynamics (RAM) simulations ${ }^{64}$ (Supplementary Note 9). To explore whether the PCS measurements on $\mathrm{C}_{2} \mathrm{~B}$ could be fit with ensemble-averaged values, we selected one representative structure from each of the 73 clusters of the chemical shift-based ensemble. For each structure, we calculated an SC166 tensor and an SC41 tensor with the PCSs measured within the SNARE complex, in Numbat. These tensors were used to calculate the $\mathrm{C}_{2} \mathrm{~B}$ PCSs for each structure. We then used MATLAB to find population weights for the 73 structures that minimize the r.m.s. deviation between the population-averaged PCSs and the experimental values. The calculations were performed separately for PCSs induced by SC166Dy and SC41Dy, thus leading to the correlations presented in Figure 4e,f.

Syt1-SNARE-complex binding assays. $1 \mathrm{D}{ }^{13} \mathrm{C}$-edited ${ }^{1} \mathrm{H}$ NMR spectra for SNARE-complex binding assays were obtained by acquiring the first trace of standard ${ }^{1} \mathrm{H}-{ }^{13} \mathrm{C}$ HSQC spectra as previously described ${ }^{34}$. Samples contained $10 \mu \mathrm{M}$ uniformly ${ }^{13} \mathrm{C}$-labeled Syt1 $\mathrm{C}_{2} \mathrm{AB}$ in $25 \mathrm{mM}$ Tris- $\mathrm{HCl}$, pH 7.4, $125 \mathrm{mM}$ KSCN, $1 \mathrm{mM} \mathrm{CaCl}_{2}, 0.5 \mathrm{mM}$ TCEP and $10 \% \mathrm{D}_{2} \mathrm{O}$, or in $50 \mathrm{mM}$ HEPES, $\mathrm{pH}$ 7.4, $100 \mathrm{mM} \mathrm{NaCl}, 1 \mathrm{mM}$ EGTA, $0.5 \mathrm{mM}$ TCEP and $10 \% \mathrm{D}_{2} \mathrm{O}$ (except for the experiments in Supplementary Figure 6a,b, which were performed with $3 \mu \mathrm{M}$ ${ }^{13} \mathrm{C}$-labeled $\mathrm{C}_{2} \mathrm{AB}$ mutants in $25 \mathrm{mM}$ Tris, $\mathrm{pH} 7.4,125 \mathrm{mM} \mathrm{NaCl}$ and $1 \mathrm{mM}$ $\mathrm{CaCl}_{2}$ ). All $\mathrm{C}_{2} \mathrm{AB}$ samples used in these assays contained the native $\mathrm{R} 398$ and R399 residues at the bottom of $\mathrm{C}_{2} \mathrm{~B}$. Unlabeled SNARE complex was titrated into the sample at the indicated concentrations. The strongest methyl resonance (SMR) intensity was measured for each point, and the natural ${ }^{13} \mathrm{C}$ abundance signal from unlabeled SNARE complex was subtracted, scaled from the SMR measurement of a sample of $20 \mu \mathrm{M}$ SNARE complex alone. Competition assays were performed similarly by acquiring $1 \mathrm{D}^{13} \mathrm{C}$-edited ${ }^{1} \mathrm{H}$ NMR spectra of $15 \mu \mathrm{M}$ SNARE complex containing ${ }^{15} \mathrm{~N}-{ }^{2} \mathrm{H}-\mathrm{ILV}-{ }^{13} \mathrm{CH}_{3}$-labeled syntaxin- 1 with different additions (as indicated in Fig. 6h,i). Liposomes contained 99\% POPC and $1 \% \mathrm{PIP}_{2}$. All titrations were performed in duplicate. No additional repeats were performed because of limited availability of the (expensive) isotopically labeled mutants and because the errors associated with these biophysical measurements are small (Fig. 6f,i and Supplementary Fig. 6c,d). Hence, the averages and s.d. values calculated from the duplicate experiments, all of which include five points, are sufficient to draw firm conclusions.

Lipid binding assays. Liposomes for binding assays were prepared with a mixture of 1-palmitoyl-2-oleoyl-sn-glycero-3-phosphocholine (POPC), 1-palmitoyl-2-oleoyl-sn-glycero-3-phosphoethanolamine (POPE), 1,2-dioleoyl-snglycero-3-phosphoethanolamine- $N$-(5-dimethylamino-1-naphthalenesulfonyl) (dansyl-DOPE), 1,2-dioleoyl-sn-glycero-3-phospho-L-serine (DOPS), cholesterol, L- $\alpha$-phosphatidylinositol (PI) and L- $\alpha$-phosphatidylinositol 4,5-diphosphate $\left(\mathrm{PIP}_{2}\right)$. For $\mathrm{Ca}^{2+}$ titrations, the mixtures contained $32 \%$ POPC, $23 \%$ POPE, $5 \%$ dansyl-DOPE, 25\% DOPS, $10 \%$ cholesterol and 5\% PI and were prepared for a stock concentration of $2 \mathrm{mM}$ total lipid. Fluorescence spectra were recorded at $25^{\circ} \mathrm{C}$ on a Photon Technology International spectrophotometer at $25{ }^{\circ} \mathrm{C}$, with excitation of the Syt 1 tryptophan fluorescence at $280 \mathrm{~nm}$ and recording of the emission from dansyl-DOPE at $528 \mathrm{~nm}$. Samples contained $0.3 \mu \mathrm{M}$ Syt1 $\mathrm{C}_{2} \mathrm{AB}$ (residues 140-421) and $100 \mu \mathrm{M}$ lipids in $50 \mathrm{mM}$ HEPES- $\mathrm{NaOH}$, $\mathrm{pH} 7.4,100 \mathrm{mM} \mathrm{NaCl}, 1 \mathrm{mM} \mathrm{MgCl} 2$ and $0.5 \mathrm{mM}$ TCEP, with the indicated concentrations of $\mathrm{Ca}^{2+}$. The FRET intensity for each point was determined by subtraction of the emission intensity of the sample in $2.5 \mathrm{mM}$ EGTA from the emission intensity at the given calcium concentration. The data were fit with Hill equations, and apparent $K_{d}$ values were calculated.

For calcium-independent binding experiments, the lipid mixture contained $40 \%$ POPC, $32 \%$ POPE, $12 \%$ DOPS, $10 \%$ cholesterol, $5 \%$ PI and $1 \%$ PIP $_{2}$. The SMR was quantified from $1 \mathrm{D}^{13} \mathrm{C}$-edited ${ }^{1} \mathrm{H}$ NMR spectra of $3 \mu \mathrm{M}^{3} \mathrm{C}$-Syt1 $\mathrm{C}_{2} \mathrm{AB}$ in $50 \mathrm{mM}$ HEPES- $\mathrm{NaOH}, \mathrm{pH} 7.4,100 \mathrm{mM} \mathrm{NaCl}, 1 \mathrm{mM} \mathrm{MgCl} 2,1 \mathrm{mM}$ EGTA, $0.5 \mathrm{mM}$ TCEP and $10 \% \mathrm{D}_{2} \mathrm{O}$, with 0 and $1,000 \mu \mathrm{M}$ total lipid concentrations.

Syt1-KO rescue experiments. Neuronal cultures were produced from WT (C57BL/6J) and Syt1-KO (also in a C57BL/6J background) mice as previously described ${ }^{65}$. Hippocampi were dissected from P0 pups, either male or female, dissociated by papain digestion and plated on Matrigel-coated glass coverslips. Neurons were cultured for 14-16 d in vitro in MEM (Gibco) supplemented with B27 (Gibco), glucose, transferrin, FBS and Ara-C (Sigma). For rescue experiments, a rat Syt1 cDNA (carrying mutations when desired) was introduced into a lentiviral construct that has been previously described ${ }^{66}$. To make viruses, human embryonic kidney 293T cells (ATCC) were cotransfected with the lentiviral vector and three packaging plasmids. Supernatant containing the viruses was collected $48 \mathrm{~h}$ after transfection and was used to infect hippocampal neuronal cultures at DIV4. Cultures were used for biochemical or physiological analyses at DIV14-16. All animal studies were approved by the Stanford University Institutional Review Board and performed in accordance with Stanford University Animal Care and Use guidelines. Although no statistical method was used to predetermine sample size, randomized experiments were performed by an experimenter blind to the status of the treatment groups.

Electrophysiological recordings in cultured neurons. Recordings were performed essentially as previously described ${ }^{67}$. The whole-cell pipette solution contained $135 \mathrm{mM} \mathrm{CsCl}, 10 \mathrm{mM}$ HEPES, 1 mM EGTA, 1 mM Na-GTP, $4 \mathrm{mM}$ $\mathrm{Mg}-\mathrm{ATP}$ and $10 \mathrm{mM}$ QX-314, $\mathrm{pH} 7.4$ (adjusted with $\mathrm{CsOH}$ ). The bath solution contained $140 \mathrm{mM} \mathrm{NaCl}, 5 \mathrm{mM} \mathrm{KCl}, 2 \mathrm{mM} \mathrm{MgCl}_{2}, 10 \mathrm{mM}$ HEPES, $10 \mathrm{mM}$ glucose, pH 7.4 (adjusted with $\mathrm{NaOH}$ ) and $2 \mathrm{mM} \mathrm{MgCl}_{2}$, unless otherwise indicated. Synaptic currents were monitored with a Multiclamp 700B amplifier (Molecular Devices). Extracellular stimuli were controlled with a Model 2100 Isolated Pulse Stimulator (A-M Systems) synchronized with the Clampex 9 or 10 dataacquisition software (Molecular Devices). Evoked synaptic responses were triggered by a bipolar electrode. GABA-R-mediated IPSCs were pharmacologically isolated with CNQX $(20 \mu \mathrm{M})$ and AP-5 $(50 \mu \mathrm{M})$ in the bath solution and recorded at a $-70 \mathrm{mV}$ holding potential. Because the intracellular solution contains high internal $\mathrm{Cl}^{-}$levels, IPSCs evoke large inward currents. mIPSCs were monitored in the presence of tetrodotoxin $(1 \mu \mathrm{M})$ in addition to the compounds listed above. Miniature events were analyzed in Clampfit 9.02 (Molecular Devices) with the template matching search and a minimal threshold of $5 \mathrm{pA}$, and each event was visually inspected for inclusion or rejection. For $\mathrm{Ca}^{2+}$ titrations, eIPSCs were measured for each cell at multiple $\mathrm{Ca}^{2+}$ concentrations starting at $2 \mathrm{mM} \mathrm{Ca}^{2+}$; this was followed by measurement of the higher, then lower, $\mathrm{Ca}^{2+}$ concentration points. For all electrophysiological experiments, the experimenter was blind to the condition genotype of the cultures analyzed.

Immunoprecipitation and quantitative immunoblotting. Cultured Syt1-KO neurons were solubilized in PBS (with $1 \mathrm{mM} \mathrm{CaCl}_{2}$ and $0.2 \%$ Triton X-100, $\mathrm{pH}$ 7.4) supplemented with protease inhibitors (Roche) for $1 \mathrm{~h}$. The lysate was cleared by centrifugation at $16,000 \mathrm{~g}$ for $10 \mathrm{~min}$ at $4{ }^{\circ} \mathrm{C}$, and immunoprecipitation 
was performed by incubation with polyclonal antibodies to syntaxin-1 (438B) that have been used previously in multiple studies (for example, refs. 68,69 ) or with preimmune sera for $1 \mathrm{~h}$ at $4{ }^{\circ} \mathrm{C}$; this was followed by incubation with $15 \mu \mathrm{l}$ of a $50 \%$ slurry of protein A-Sepharose beads (GE Healthcare) for $2 \mathrm{~h}$ at $4{ }^{\circ} \mathrm{C}$. Beads were washed $4 \times$ with $1 \mathrm{ml}$ extraction buffer, and bound proteins were eluted with $2 \times$ SDS sample buffer containing $100 \mathrm{~mm}$ DTT and were boiled for $20 \mathrm{~min}$ at $100^{\circ} \mathrm{C}$.

Coprecipitated proteins were separated by SDS-PAGE; this was followed by detection with monoclonal antibodies against rat Syt1 (604.4, Synaptic Systems) and synaptobrevin-2 (clone 69.1, Synaptic Systems) (validation provided by the manufacturer). To allow for quantitative detection, dye-conjugated secondary antibodies were used (IRDye 800CW donkey anti-mouse IgG, Li-cor), membranes were scanned in an Odyssey scanner (Li-cor), and quantification was performed with Image Studio software (Li-cor).

59. Delaglio, F. et al. NMRPipe: a multidimensional spectral processing system based on UNIX pipes. J. Biomol. NMR 6, 277-293 (1995).

60. Johnson, B.A. \& Blevins, R.A. NMR View: a computer-program for the visualization and analysis of NMR data. J. Biomol. NMR 4, 603-614 (1994).
61. Shao, X., Fernandez, I., Sudhof, T.C. \& Rizo, J. Solution structures of the $\mathrm{Ca}^{2+}$-free and $\mathrm{Ca}^{2+}$-bound $\mathrm{C} 2 \mathrm{~A}$ domain of synaptotagmin I: does $\mathrm{Ca}^{2+}$ induce a conformational change? Biochemistry 37, 16106-16115 (1998).

62. Chen, X., Tang, J., Sudhof, T.C. \& Rizo, J. Are neuronal SNARE proteins $\mathrm{Ca}^{2+}$ sensors? J. Mol. Biol. 347, 145-158 (2005).

63. Schmitz, C., Stanton-Cook, M.J., Su, X.C., Otting, G. \& Huber, T. Numbat: an interactive software tool for fitting Deltachi-tensors to molecular coordinates using pseudocontact shifts. J. Biomol. NMR 41, 179-189 (2008).

64. Camilloni, C. \& Vendruscolo, M. Statistical mechanics of the denatured state of a protein using replica-averaged metadynamics. J. Am. Chem. Soc. 136, 8982-8991 (2014).

65. Maximov, A., Pang, Z.P., Tervo, D.G. \& Sudhof, T.C. Monitoring synaptic transmission in primary neuronal cultures using local extracellular stimulation. J. Neurosci. Methods 161, 75-87 (2007).

66. Pang, Z.P., Cao, P., Xu, W. \& Sudhof, T.C. Calmodulin controls synaptic strength via presynaptic activation of calmodulin kinase II. J. Neurosci. 30, 4132-4142 (2010).

67. Bacaj, T. et al. Synaptotagmin-1 and synaptotagmin-7 trigger synchronous and asynchronous phases of neurotransmitter release. Neuron 80, 947-959 (2013).

68. Zhou, P. et al. Syntaxin-1 N-peptide and Habc-domain perform distinct essential functions in synaptic vesicle fusion. EMBO J. 32, 159-171 (2013).

69. Sharma, M. et al. CSP $\alpha$ knockout causes neurodegeneration by impairing SNAP-25 function. EMBO J. 31, 829-841 (2012). 
Copyright of Nature Structural \& Molecular Biology is the property of Nature Publishing Group and its content may not be copied or emailed to multiple sites or posted to a listserv without the copyright holder's express written permission. However, users may print, download, or email articles for individual use. 